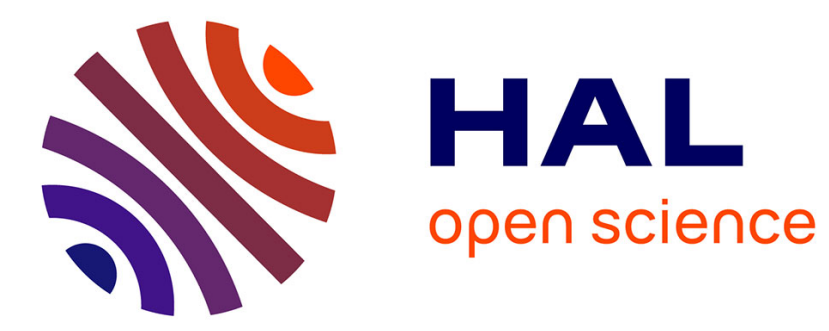

\title{
Transfert d'énergie inductive par plaques métalliques en mouvement rapide
}

\author{
F. Rioux-Damidau
}

\section{To cite this version:}

F. Rioux-Damidau. Transfert d'énergie inductive par plaques métalliques en mouvement rapide. Revue de Physique Appliquée, 1977, 12 (1), pp.47-60. 10.1051/rphysap:0197700120104700 . jpa00244119

\section{HAL Id: jpa-00244119 https://hal.science/jpa-00244119}

Submitted on 1 Jan 1977

HAL is a multi-disciplinary open access archive for the deposit and dissemination of scientific research documents, whether they are published or not. The documents may come from teaching and research institutions in France or abroad, or from public or private research centers.
L'archive ouverte pluridisciplinaire HAL, est destinée au dépôt et à la diffusion de documents scientifiques de niveau recherche, publiés ou non, émanant des établissements d'enseignement et de recherche français ou étrangers, des laboratoires publics ou privés. 


\begin{abstract}
Classification
Physics Abstracts

0.660

\section{TRANSFERT D'ÉNERGIE INDUCTIVE PAR PLAQUES MÉTALLIQUES EN MOUVEMENT RAPIDE}

\author{
F. RIOUX-DAMIDAU \\ Laboratoire de Physique des Plasmas-Groupe Fusion \\ Université Paris-Sud, 91405 Orsay, France
}

(Reçu le 10 juin 1976, accepté le 28 juillet 1976)

\begin{abstract}
Résumé. - Le transfert de l'énergie stockée dans une inductance vers une charge inductive nécessite l'utilisation d'un disjoncteur composé d'un interrupteur mécanique et d'un système absorbeur (usuellement des feuilles explosantes) capable d'absorber une forte énergie. Le rendement maximal habituel du transfert est de $25 \%$. On présente ici un nouveau type d'absorbeur, utilisant des plaques métalliques en mouvement rapide, capable d'effectuer le transfert avec un rendement élevé $(60-70 \%)$. Il est adapté aux fortes énergies $(>1 \mathrm{MJ})$ et aux temps courts $(10-100 \mu \mathrm{s})$. Son utilisation entraîne moins de destructions que celle des feuilles.
\end{abstract}

\begin{abstract}
In order to transfer the energy stored in a coil to an inductive load one needs a circuit-breaker consisting of a mechanical switch and an absorbing device (usually an exploding foil) capable of absorbing a high energy. The usual maximum efficiency of the transfert is $25 \%$. We describe here a new type of absorber with rapidly moving metallic plates which can transfer the energy with a high efficiency $(60-70 \%)$. It is convenient for high energies ( $>1 \mathrm{MJ})$ and for small delivery times $(10-100 \mu \mathrm{s})$. Its use causes less destruction than with the foils.
\end{abstract}

1. Introduction. - Pour diverses utilisations et en particulier pour les expériences de fusion thermonucléaire contrôlée du type à confinement mixte $[1,2$, $3,4]$, il est nécessaire de réaliser des sources délivrant une énergie importante en un temps court (quelque $10^{8} \mathrm{~J}$ en quelques $\mu \mathrm{s}$ ).

L'utilisation de génératrices homopolaires ou hétéropolaires spéciales, susceptibles de convertir l'énergie cinétique d'un volant en énergie électromagnétique que l'on stocke dans une bobine, devrait fournir une source primaire très bon marché $[5,6,7]$. Mais, pour transférer cette énergie sur la charge, il faut disposer de disjoncteurs rapides. Ces derniers seront formés d'un ouvreur mécanique qui laissera passer le courant pendant toute la durée du stockage puis dérivera lors de son fonctionnement, le courant sur un absorbeur capable de dissiper l'énergie requise par le processus de transfert [8].

Usuellement, cet absorbeur est constitué par une feuille explosante [9]. A fort niveau d'énergie, le problème de l'emploi de telles feuilles risque de devenir un obstacle important. Pour assurer une explosion rapide, les feuilles doivent être fines; leur surface devient donc grande (pour absorber $100 \mathrm{MJ}$, il faut plus de $10 \mathrm{~m}^{2}$ de feuille de cuivre de $0,1 \mathrm{~mm}$ d'épaisseur). La mise en place devient difficile d'autant plus qu'il faut remplacer les isolants pollués dont le volume est très grand; de plus les électrodes se détériorent progressivement. Enfin et surtout le choc brisant créé par cette explosion ne peut être soutenu que par une enceinte ayant une masse considérable.

Nous proposons ici un système qui pourrait jouer ce rôle d'absorbeur sans engendrer de choc tout en ayant un volume réduit.

2. Principe du système. -2.1 Fonctionnement. Le schéma de principe du montage est donné par la figure 1. Pour faciliter la description de son fonctionnement, nous supposons que tous ses éléments ont la même largeur $\mathcal{L}$, laquelle est très grande.

Les deux bobines, de stockage $\mathfrak{B}_{1}$ et de charge $\mathfrak{B}_{2}$, ont pour surface $S_{1}$ et $S_{2}$. L'élément central, qui est l'absorbeur, est composé d'une enceinte métallique et d'une plaque conductrice $P$. Lorsque la plaque est dans une position quelconque, la surface de l'enceinte à gauche de $\mathrm{P}$ vaut $s_{1}$ et celle à droite vaut $s_{2}$, la surface totale de l'enceinte valant $s=s_{1}+s_{2}$.

Durant la phase initiale de stockage de l'énergie, l'ouvreur $\mathrm{D}$ est fermé, le champ régnant dans $\mathfrak{B}_{1}$ vaut $B_{1 \mathrm{i}}$, la plaque $\mathbf{P}$ est complètement à gauche, $s_{1}=0, s_{2}=s$, l'inductance du circuit compris entre $\mathrm{D}$ et $\mathrm{P}$ est très petite. Un faible champ magnétique $B_{2 \mathrm{i}}$ est créé dans $s_{2}$ et $S_{2}$ c'est-à-dire dans l'enceinte 
et $\mathfrak{B}_{2}$. Si l'on considère les énergies par unité de largeur, celle de $\mathfrak{B}_{1 \mathrm{i}}$ qui est l'énergie stockée vaut

$$
W_{\mathrm{s}}=B_{1 \mathrm{i}}^{2} S_{1} / 2 \mu_{0}
$$

et celle de $B_{2 \mathrm{i}}$, que nous appelons énergie de commande, vaut

$$
W_{\mathrm{c}}=B_{2 \mathrm{i}}^{2}\left(S_{2}+s_{2}\right) / 2 \mu_{0}
$$

on a $W_{\mathrm{s}} \gg W_{\mathrm{c}}$.

Lorsque l'on ouvre $\mathrm{D}$, l'induction magnétique $B_{1, \mathrm{i}} \gg B_{2, \mathrm{i}}$ accélère la plaque. Les flux magnétiques $\Phi_{1}=B_{1}\left(S_{1}+s_{1}\right)$ et $\Phi_{2}=B_{2}\left(S_{2}+s_{2}\right)$ étant conservés à gauche et à droite de $\mathrm{P}$, l'induction $B_{1}$ diminue, l'induction $B_{2}$ augmente, l'énergie

$$
W_{1}=\Phi_{1}^{2} / 2 \mu_{0}\left(S_{1}+s_{1}\right)
$$

de $B_{1}$ diminue, l'énergie $W_{2}=\Phi_{2}^{2} / 2 \mu_{0}\left(S_{2}+s_{2}\right)$ de $B_{2}$ augmente. Lorsque $B_{2}$ devient supérieur à $B_{1}$, $\mathrm{P}$ est ralentie et enfin arrêtée à moins qu'elle ne vienne auparavant heurter la paroi de l'enceinte.

Dans un fonctionnement bien adapté, la plaque arrive sur la paroi avec une vitesse nulle. En fin de course, on a $B_{1}=B_{1 \mathrm{f}}$ et $B_{2}=B_{2 \mathrm{f}}$.

Au cours du fonctionnement de cet absorbeur, une partie de l'énergie stockée se transforme donc en énergie cinétique qui elle-même passe dans la charge sous forme d'énergie magnétique. Un transfert a bien été effectué, dont nous allons étudier les performances.

Pour éviter tout choc sur la paroi de l'enceinte, il est préférable d'utiliser un montage à deux plaques tel que celui représenté par la figure 2. Les plaques viennent alors se frapper l'une contre l'autre. L'étude théorique des deux montages est bien évidemment la même, avec les correspondances de dimensions indiquées sur les figures 1 et 2 .

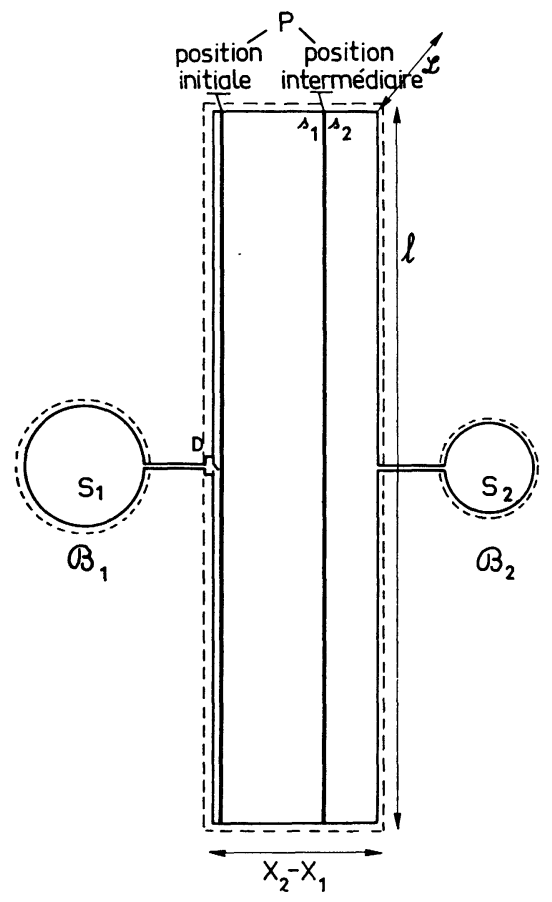

Frg. 1. - Schéma de l'absorbeur à une plaque.

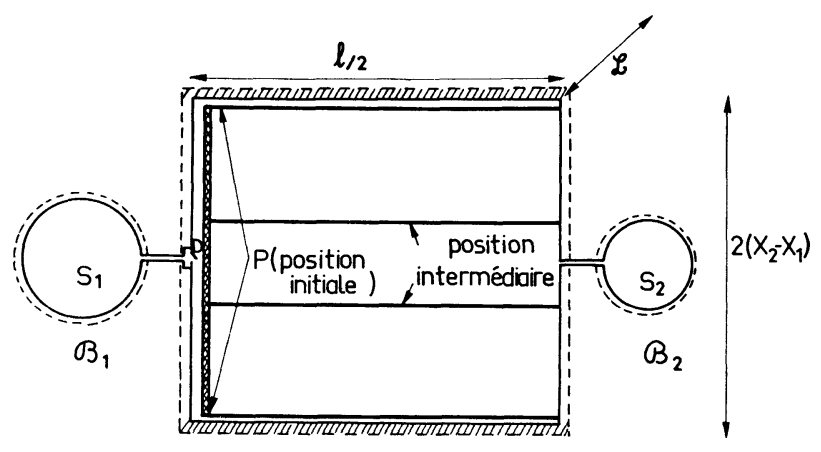

FIG. 2. - Schéma de l'absorbeur à deux plaques.

2.2 Rendement en ABSENCE De PerTes. - Pour simplifier l'étude du système, nous adopterons le schéma équivalent de la figure 3. Dans une enceinte rectangulaire, de hauteur $l$ et de longueur $X$ est placée une plaque $P$. La largeur $\mathcal{L}$ est suffisamment grande pour que les distributions de champ magnétique soient les mêmes que dans un système infiniment large.

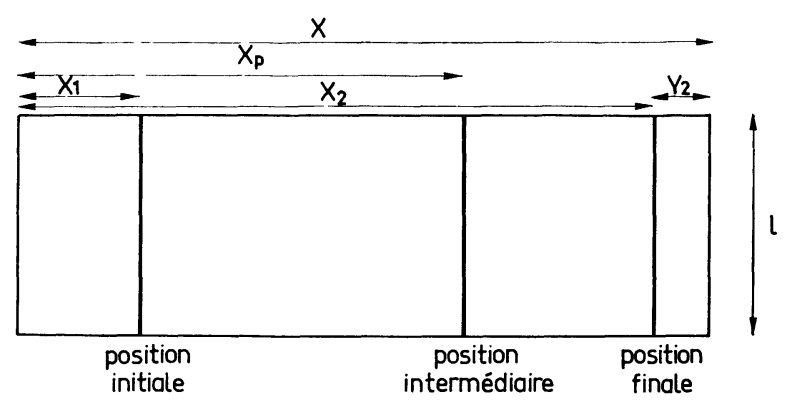

Fig. 3. - Schéma du principe de fonctionnement de l'absorbeur.

Le flux global vaut $\Phi_{0}$; il est partagé entre les flux

$$
\begin{aligned}
& \Phi_{1}=\varphi_{1} \Phi_{0} \text { à gauche de } \mathbf{P} \\
& \Phi_{2}=\varphi_{2} \Phi_{0} \text { à droite de } \mathrm{P} \\
& \left(\varphi_{1}+\varphi_{2}=1\right) .
\end{aligned}
$$

En admettant que les parois de l'enceinte et la plaque sont infiniment conductrices, ce que nous ferons dans cette première étape de l'étude en absence de pertes, ces trois flux restent séparément conservatifs au cours du mouvement de P. $\varphi_{1}$ et $\varphi_{2}$ sont donc constants.

Initialement, la plaque $\mathrm{P}$ occupe la position $X_{1}$ avec une vitesse nulle. Ensuite, lorsqu'on laisse agir l'induction initiale $B_{1, i}, P$ s'accélère puis ralentit comme décrit plus haut, sa position étant repérée par la cote $X_{\mathrm{P}}$ et enfin s'arrête au point $X_{2}$. Nous poserons

$$
\begin{aligned}
& x_{1}=\frac{X_{1}}{X}, \quad x_{2}=\frac{X_{2}}{X}, \quad x_{\mathrm{P}}=\frac{X_{\mathrm{P}}}{X}, \ldots \\
& Y_{2}=X-X_{2}, \quad Y_{\mathrm{P}}=X-X_{\mathrm{P}}, \ldots \\
& y_{2}=\frac{Y_{2}}{X}, \ldots
\end{aligned}
$$


Considérons les énergies mises en jeu (toujours par unité de largeur). Initialement l'énergie stockée est $W_{\mathrm{s}}=\Phi_{1}^{2} / 2 \mu_{0} X_{1} l$, l'énergie de commande $W_{\mathrm{c}}=\Phi_{2}^{2} / 2 \mu_{0} Y_{1} l$ et l'énergie globale vaut donc

$$
W_{0}=W_{\mathrm{c}}+W_{\mathrm{s}}=\frac{\Phi_{0}^{2}}{2 \mu_{0} l}\left(\frac{\varphi_{1}^{2}}{X_{1}}+\frac{\varphi_{2}^{2}}{Y_{1}}\right) .
$$

Lorsque la plaque est au point $X_{\mathrm{P}}$, elle a une énergie cinétique $W_{\mathrm{p}}$. Les énergies magnétiques à gauche et à droite de $\mathrm{P}$, compte tenu de ce que les flux sont conservatifs, valent $\Phi_{1}^{2} / 2 \mu_{0} X_{\mathrm{P}} l$ et $\Phi_{2}^{2} / 2 \mu_{0} \mathrm{Y}_{\mathrm{P}} l$.

L'énergie magnétique globale vaut :

$$
W_{\mathrm{M}}=\frac{\Phi_{0}^{2}}{2 \mu_{0} l}\left(\frac{\varphi_{1}^{2}}{X_{\mathbf{P}}}+\frac{\varphi_{2}^{2}}{X-X_{\mathrm{P}}}\right) .
$$

L'énergie totale du système étant conservative, on a :

$$
W_{0}=W_{\mathbf{M}}+W_{\mathbf{P}}=\text { cte } .
$$

La figure 4 représente $W_{\mathrm{M}}$ en fonction de la position de la plaque. On y lit aussi immédiatement l'énergie

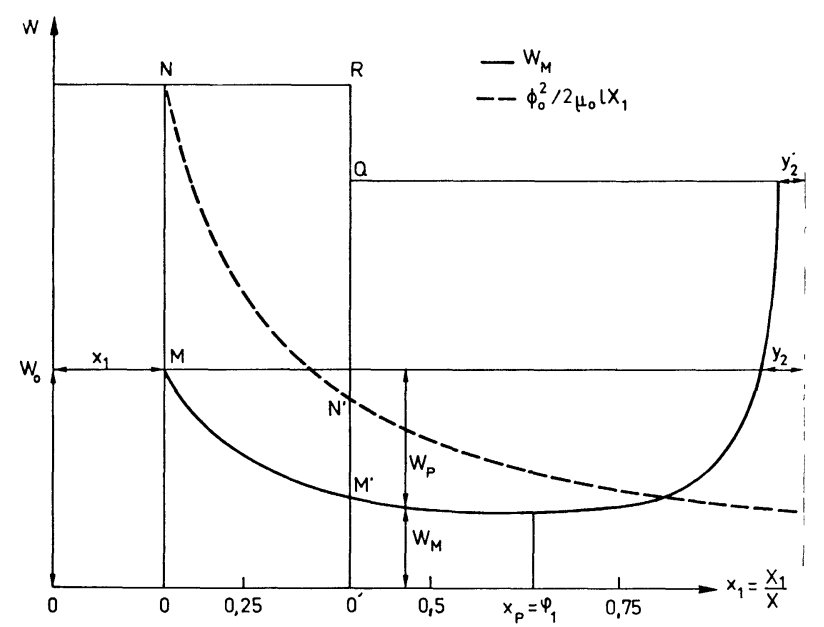

Fig. 4. - Variation de l'énergie magnétique au cours du mouvement de la plaque.

cinétique $W_{\mathrm{P}} . W_{\mathrm{M}}$ passe par un minimum (égal à $\Phi_{0}^{2} / 2 \mu_{0} X l$ ) pour $X_{\mathbf{P}}=\varphi_{1} X$ (et $Y_{\mathbf{P}}=\varphi_{2} \mathrm{X}$ ). A ce moment, les inductions magnétiques

$$
B_{1}=\frac{\varphi_{1} \Phi_{0}}{X_{\mathrm{P}} l} \quad \text { et } \quad B_{2}=\frac{\varphi_{2} \Phi_{0}}{Y_{\mathrm{P}} l}
$$

sont égales et valent $\frac{\Phi_{0}}{X l}$.

Le point $X_{2}$ d'arrêt de la plaque est donné à partir de (3), (4) et (5) par :

$$
\frac{\varphi_{1}^{2}}{X_{2}}+\frac{\varphi_{2}^{2}}{X-X_{2}}=\frac{\varphi_{1}^{2}}{X_{1}}+\frac{\varphi_{2}^{2}}{X-X_{1}}
$$

soit

$$
\frac{\varphi_{1}^{2}}{\varphi_{2}^{2}}=\frac{X_{1} X_{2}}{\left(X-X_{1}\right)\left(X-X_{2}\right)}=\frac{X_{1} X_{2}}{Y_{1} Y_{2}}
$$

Le rendement du transfert vaut alors :

$r_{0}=\frac{\text { énergie finale } W_{2} \text { dans } \mathscr{B}_{2}}{\text { énergie initiale, globale, } W_{0}}=\frac{\varphi_{2}^{2} / Y_{2}}{\frac{\varphi_{1}^{2}}{X_{1}}+\frac{\varphi_{2}^{2}}{Y_{1}}}=\frac{Y_{1}}{X}$

$r_{0}=1-x_{1}$

Le rapport entre l'énergie de commande $W_{\mathrm{c}}$ et l'énergie stockée $W_{\mathrm{s}}$ vaut :

$$
C=\frac{\varphi_{2}^{2}}{Y_{1}} \cdot \frac{X_{1}}{\varphi_{1}^{2}}=\frac{Y_{2}}{X_{2}}=\frac{1-x_{2}}{x_{2}}=\frac{y_{2}}{1-y_{2}} .
$$

Pour créer l'énergie de commande on utilisera en général un système annexe qui, dans la majeure partie des cas, aura une énergie initiale $2 W_{\mathrm{c}}$. On sera donc amené à considérer le rendement

$$
\begin{aligned}
& r=\frac{W_{2}}{W_{0}+W_{\mathrm{c}}}=\frac{\varphi_{2}^{2} / Y_{2}}{\frac{\varphi_{1}^{2}}{X_{1}}(1+2 C)} \\
& r=\frac{X-X_{1}}{X+Y_{2}}=\frac{1-x_{1}}{1+y_{2}} .
\end{aligned}
$$

On a donc intérêt à ce que $x_{1}$ et $y_{2}$ soient petits, c'est-à-dire à ce que les bobines de stockage et de charge soient petites comparées à l'absorbeur, pour que les rendements soient élevés et l'énergie de commande petite. Ces rendements peuvent être excellents. Par exemple si $x_{1}=0,1$ et $y_{2}=0,04$ on a $r_{0}=90 \%$ et $r=86 \%$ tandis que l'énergie de commande n'est que $4 \%$ de l'énergie stockée (on a alors $\varphi_{1}=0,62$ et $\left.\varphi_{2}=0,38\right)$. En général, $y_{2}$ sera tel que $y_{2}<x_{1}$ pour que l'énergie de commande soit vraiment faible.

Ce système peut donc avoir, à priori, un bon rendement. Deux problèmes essentiels se posent maintenant :

- peut-on extraire l'énergie de commande de l'énergie stockée ou faut-il une source extérieure ?

- comment les pertes modifient-elles le rendement théorique?

Nous allons les examiner successivement.

3. Obtention de l'énergie de commande à partir de l'énergie initiale. - 3.1 DIFFUSION DE L'ÉNERGIE INITIALE. - Supposons qu'initialement le flux dans $\Re_{1}$ soit égal à $\Phi_{0}$ et celui à droite de $P$ nul. Au début du mouvement de $P$, on laisse diffuser très rapidement une partie de $\Phi_{0}$ vers la droite (par exemple en mettant transitoirement une résistance en série avec $P$ ), puis on arrête ce phénomène (en court-circuitant la résistance). Les flux ont alors les valeurs $\Phi_{1}$ et $\Phi_{2}$. L'énergie initiale

$$
\frac{\Phi_{0}^{2}}{2 \mu_{0} l X_{1}},
$$

représentée par $O N$ sur la figure 4, est devenue

$$
\frac{1}{2 \mu_{0} l}\left(\frac{\Phi_{1}^{2}}{X_{1}}+\frac{\Phi_{2}^{2}}{Y_{1}}\right) \text {, }
$$


représentée par $O M$. L'énergie perdue $N M$ (par dissipation par effet Joule dans la résistance) est importante comme on le voit sur la figure. En fait, par ce procédé, on transfère de l'énergie d'une inductance assez faible (proportionnelle à $X_{1}$ ) vers une autre plus grande (proportionnelle à $Y_{1}$ ); l'énergie perdue dans le système qui sert à effectuer le transfert est alors très importante, comme lorsqu'on utilise un disjoncteur dans des conditions semblables.

On peut alors envisager de procéder autrement. Au lieu d'effectuer le transfert de flux au point $X_{1}$, on laisse tout d'abord la plaque acquérir une certaine vitesse, le flux $\Phi_{0}$ restant intégralement concentré dans $\mathfrak{B}_{1}$ puis, en un point plus lointain, on effectue le transfert; l'énergie magnétique passe (Fig. 4) de la valeur $O^{\prime} N^{\prime}$ à la valeur $O^{\prime} M^{\prime}$, l'énergie perdue étant $N^{\prime} M^{\prime}$, l'énergie totale devient $O^{\prime} Q$ avec $R Q=N^{\prime} M^{\prime}$ l'énergie cinétique restant conservée $\left(M^{\prime} Q=N^{\prime} R\right)$. On constate que l'énergie perdue est beaucoup plus petite que si on effectue la diffusion au départ $\left(M^{\prime} N^{\prime}<M N\right)$ et que l'énergie totale du système est plus grande $\left(O^{\prime} Q>O M\right)$ bien qu'étant parti de la même énergie initiale $O N$. D'autre part, la distance d'arrêt $Y_{2}^{\prime}$ devient plus petite; le flux $\Phi_{2}$ est donc plus comprimé et l'énergie finale utilisable plus grande. Pour cet.e opération, il est évident que le meilleur point de diffusion correspond au minimum de la courbe donnant $W_{\mathrm{M}}$ c'est-à-dire au point $x_{\mathrm{P}}=\varphi_{1}$. La diffusion tend alors à égaliser les inductions de part et d'autre de $\mathrm{P}$ puisque, à la fin, on doit avoir $B_{2}=B_{1}$ (elle ne peut avoir lieu en un point plus lointain car il faudrait obtenir $B_{2}>B_{1}$ ). En ce point $x_{\mathrm{P}}=\varphi_{1}$, les conditions d'un bon rendement sont donc réalisées.

La figure 5 représente le rendement en fonction de $\varphi_{1}$ et pour diverses valeurs de $x_{1}$ dans trois conditions différentes :

- lorsque la diffusion a lieu au point de départ de la plaque $\left(r_{\mathrm{D}}\right)$

- lorsqu'elle est effectuée au point $x_{\mathrm{P}}=\varphi_{1}\left(r_{\mathrm{m}}\right)$

- lorsque l'énergie de commande est fournie au départ par un procédé non précisé ayant une énergie double ; $r$ est alors donné par (9).

On constate que $r$ et $r_{\mathrm{m}}$ sont peu différents et bons mais que $r_{\mathrm{D}}$ est effectivement très mauvais. La méthode de la diffusion en $x_{\mathrm{P}}=\varphi_{1}$ semble donc à priori possible.

Il faut cependant remarquer que l'on doit laisser diffuser l'énergie alors que la plaque occupe une position précise et est en pleine vitesse sinon le rendement diminue beaucoup. D'autre part, le volume final de $\mathfrak{B}_{2}$ étant plus petit que dans le cas où l'énergie de commande est présente à droite de $\mathbf{P}$ dès le départ, les pertes dues aux défauts des plaques seront plus sensibles (cf. § 6). Cette méthode ne semble donc pas très prometteuse.

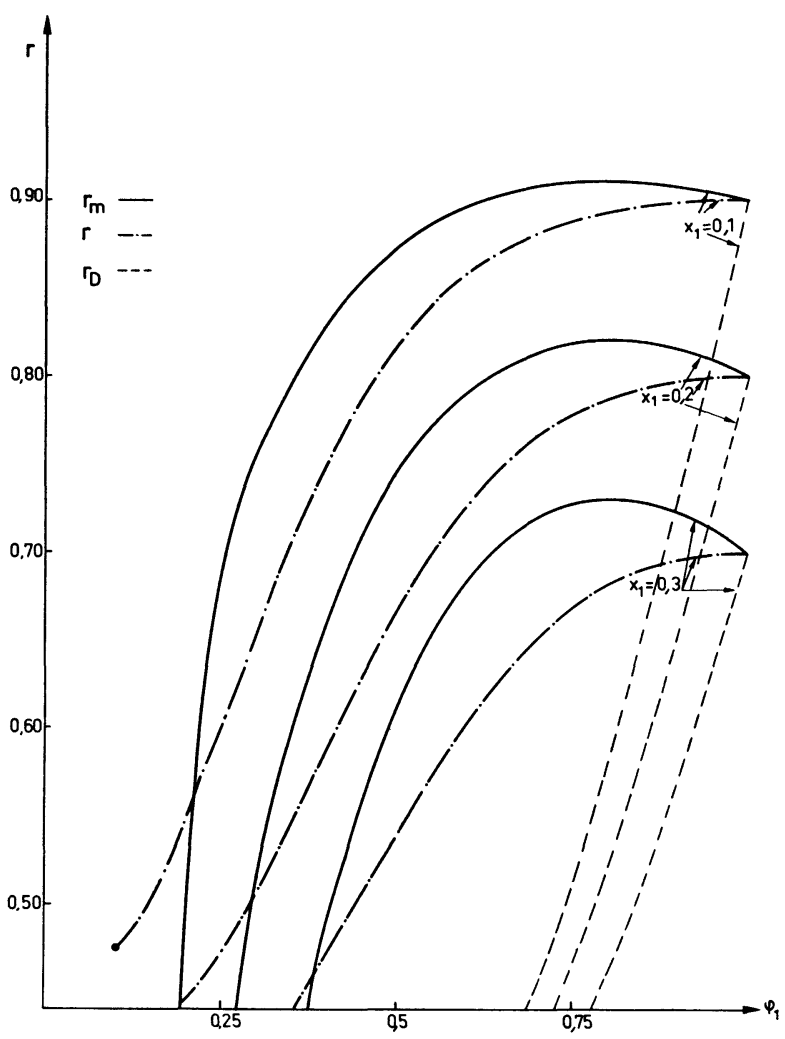

Fig. 5. - Rendement du transfert suivant les conditions de fonctionnement de l'absorbeur.

3.2 MONTAGE AVEC ENCEINTE EXTÉRIEURE SUPPLÉMENTAIRE. - Le problème qui se pose est donc le suivant : comment extraire une partie du flux de la bobine de stockage pour le mettre à droite de la plaque de façon simple et avec un bon rendement. Nous proposons maintenant un montage, un peu plus compliqué technologiquement que le précédent, mais qui permet le transfert de flux ; le schéma de principe en est donné figure 6. Il diffère essentiellement du précédent par la présence d'une enceinte entourant l'élément central et la bobine de récupération $\mathfrak{B}_{2}$. Le fonctionnement est le suivant (Fig. 6) :

- Etape I: l'enceinte est en circuit ouvert au départ. Le flux $\Phi_{0}$ règne dans $\mathscr{B}_{1}$ et un flux nul dans l'enceinte. La plaque, initialement au repos, commence à s'accélérer.

\section{- Etape II : la plaque étant en position}

$$
X_{\mathrm{a}}=X_{1}+Z_{\mathrm{a}},
$$

on ferme le circuit de l'enceinte. Le flux total dans l'enceinte vaut $\Phi_{\mathrm{a}}$ et va désormais garder cette valeur. On a :

$$
\frac{\Phi_{0}-\Phi_{\mathrm{a}}}{X_{1}}=\frac{\Phi_{\mathrm{a}}}{Z_{\mathrm{a}}}
$$

ce qui donne :

$$
\Phi_{\mathrm{a}}=\frac{Z_{\mathrm{a}} \Phi_{0}}{Z_{\mathrm{a}}+X_{1}}
$$


I

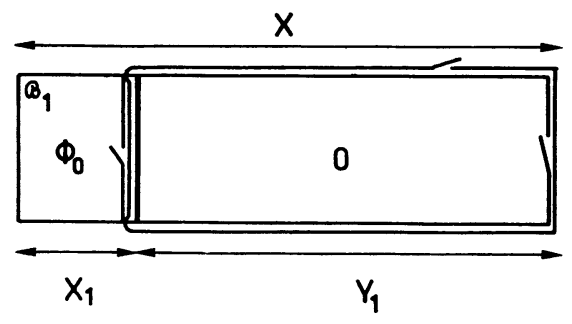

II

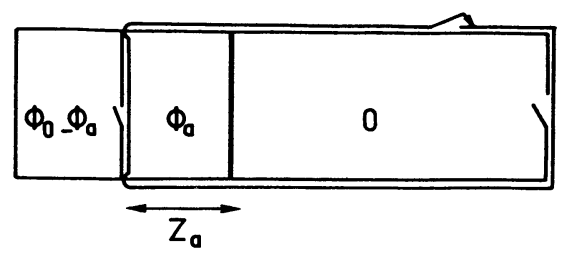

III

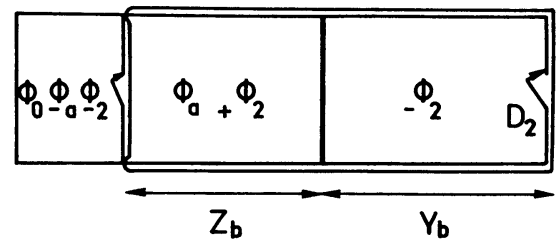

IV

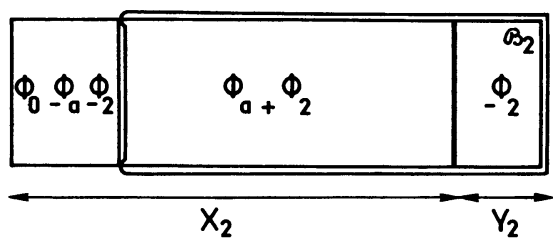

Fig. 6. - Montage avec enceinte extérieure.

- Etape III : la plaque étant en position

$$
X_{\mathrm{b}}=X_{1}+Z_{\mathrm{b}} \text {, }
$$

on court-circuite la bobine $\mathfrak{B}_{1}$. Les flux valent respectivement $\Phi_{0}-\Phi_{\mathrm{a}}-\Phi_{2}$ dans $\Re_{1}$ et, dans l'enceinte, $\Phi_{\mathrm{a}}+\Phi_{2}$ à gauche de $\mathrm{P}$ et $\left(-\Phi_{2}\right)$ à droite de $\mathrm{P}$. Ils sont, à partir de maintenant, séparément conservatifs et vérifient :

$$
\frac{\Phi_{0}-\Phi_{\mathrm{a}}-\Phi_{2}}{X_{1}}=\frac{\Phi_{\mathrm{a}}+\Phi_{2}}{Z_{\mathrm{b}}}=\frac{\Phi_{2}}{Y_{\mathrm{b}}}
$$

ce qui donne :

$$
\Phi_{2}=\frac{X_{\mathrm{a}} Y_{\mathrm{b}}\left(Z_{\mathrm{b}}-Z_{\mathrm{a}}\right)}{X_{\mathrm{a}}\left[X_{1} Z_{\mathrm{b}}+X_{1} Y_{\mathrm{b}}+Z_{\mathrm{b}} Y_{\mathrm{b}}\right]} \Phi_{0} .
$$

On peut aussi, si on le désire, fermer le conjoncteur $D_{2}$, ce qui évitera de comprimer le flux compris entre les parois de l'élément central et de la bobine $\mathfrak{B}_{2}$ et celles de l'enceinte.

- Etape IV : la plaque s'arrête en un point $X_{2}$.
La valeur de $X_{2}$ (ou $Y_{2}$ ) s'obtient à partir de l'équation de conservation de l'énergie

$$
\frac{\Phi_{0}^{2}}{X_{1}}=\frac{\left(\Phi_{0}-\Phi_{\mathrm{a}}-\Phi_{2}\right)^{2}}{X_{1}}+\frac{\left(\Phi_{\mathrm{a}}+\Phi_{2}\right)^{2}}{X_{2}-X_{1}}+\frac{\Phi_{2}^{2}}{Y_{2}} .
$$

L'ensemble de ces équations nous a permis de calculer le rendement $r_{\mathrm{e}}$ qui dépend de $X_{1}, X_{\mathrm{a}}$ et $X_{\mathrm{b}}$. Nous avons constaté que, pour $X_{1}$ et $X_{\mathrm{a}}$ donnés, $r_{\mathrm{e}}$ passe par un maximum très plat pour $X_{\mathrm{b}} \simeq 0,5$ à $0,6 X$. Nous avons alors choisi la valeur de $X_{b}$ qui conduit à la valeur maximale de $r_{\mathrm{e}}$ et nous avons obtenu : $r_{\mathrm{e}}=r_{\mathrm{e}}\left(X_{1}, X_{\mathrm{a}}\right) \quad y_{2}=y_{2}\left(X_{1}, X_{\mathrm{a}}\right)$ d'où nous avons déduit

$$
r_{\mathrm{e}}=r_{\mathrm{e}}\left(X_{1}, Y_{2}\right) .
$$

La figure 7 représente $r_{\mathrm{e}}$ en fonction de $Y_{1} / Y_{2}$ pour diverses valeurs de $X_{1} / X-X_{1}$ c'est-à-dire du

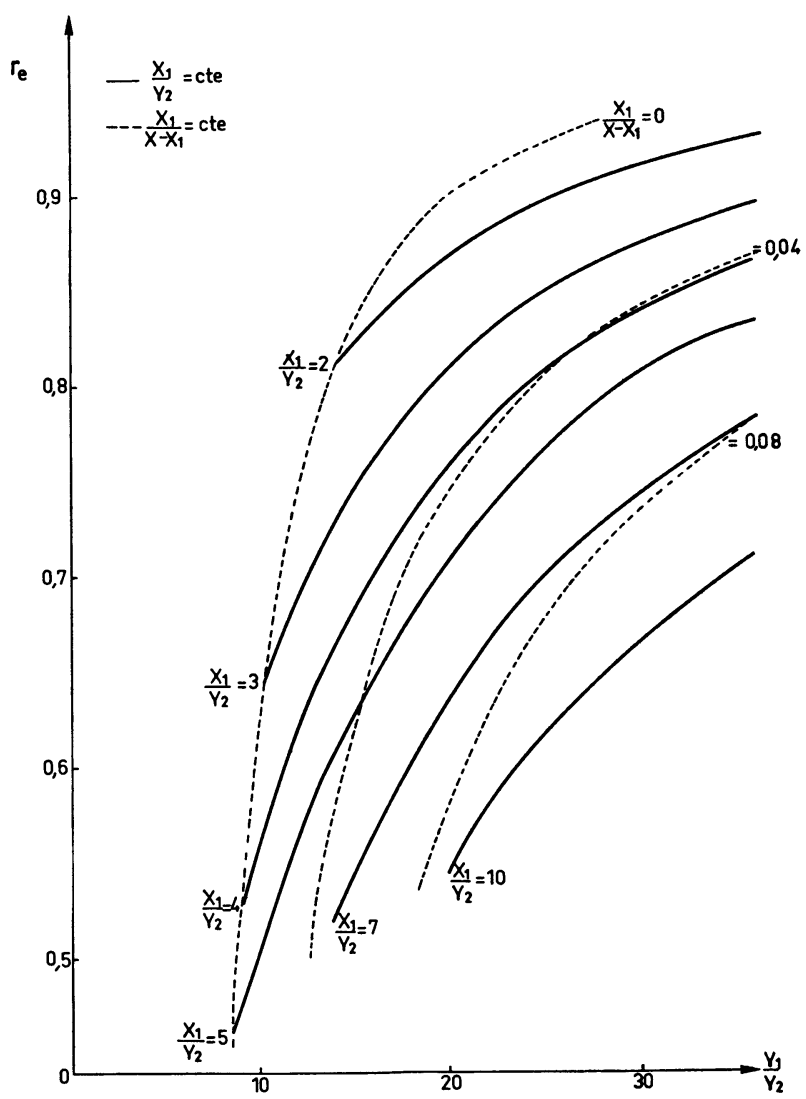

Fig. 7. - Rendement du montage avec enceinte extérieure.

rapport entre le volume de la bobine $\mathfrak{B}_{1}$ et celui de l'ensemble absorbeur + bobine $\mathfrak{B}_{2}$ et pour diverses valeurs de $X_{1} / Y_{2}$ c'est-à-dire du rapport des volumes de $\mathfrak{B}_{1}$ et $\mathfrak{B}_{2}$. Le rendement $r_{\mathrm{e}}$ peut être bon mais décroît si ces deux rapports augmentent. Un bon fonctionnement nécessite

$$
\frac{X_{1}}{X-X_{1}} \lesssim 0,05 \text { et } \frac{X_{1}}{Y_{2}} \lesssim 5 .
$$


On peut facilement voir que $r_{\mathrm{e}}$ est sensiblement donné par :

$$
r_{\mathrm{e}} \simeq \frac{X-2 X_{1}}{X-X_{1}+Y_{2}} .
$$

La méthode décrite ici peut permettre de prendre l'énergie de commande sur l'énergie initiale et d'obtenir un bon rendement de transfert (par exemple si $x_{1}=0,13, \quad y_{2}=0,044, \quad z_{\mathrm{a}}=0,018, \quad z_{\mathrm{b}}=0,435$, $r_{\mathrm{e}}=81 \%$ et $\left.\Phi_{\mathrm{a}}=0.13 \Phi_{0}, \Phi_{2}=0.52 \Phi_{0}\right)$. Elle est cependant un peu complexe. On sera donc amené, suivant les cas, à choisir entre cette solution et celle d'une source extérieure.

4. Dynamique de la plaque. - Pour préciser les performances du système, il nous faut maintenant évaluer les pertes qui ont plusieurs origines :

- diffusion du champ magnétique au travers de $P$ au cours de son mouvement

- effet joule dans $\mathbf{P}$ et dans les parois de l'enceinte ;

- champ piégé dans les épaisseurs de peau diverses;

- défauts de planéité des plaques, qui s'accroissent au cours de leur mouvement.

Cette étude, qui nécessite en premier lieu la connaissance de la dynamique de la plaque, sera effectuée ici dans le cas simple du premier montage sans enceinte extérieure. Une étude menée par ailleurs nous a montré que les résultats obtenus restaient globalement valables.

Considérons donc la plaque du montage de la figure 3. Son épaisseur est $e$, sa hauteur $l$, sa masse spécifique $\bar{\omega}$. On obtient facilement son énergie cinétique $W_{\mathbf{P}}$ (toujours pour une largeur unité du montage) en écrivant la conservation de l'énergie totale :

$$
W_{\mathbf{P}}=W_{0}-W_{\mathbf{M}} \text {. }
$$

D'après (3) et (4), ceci donne :

$W_{\mathrm{P}}=\frac{1}{2} \bar{\omega} v^{2} e l=\frac{\Phi_{0}^{2}}{2 \mu_{0} l}\left(\frac{\varphi_{1}^{2}}{X_{1}}-\frac{\varphi_{1}^{2}}{X_{\mathrm{P}}}+\frac{\varphi_{2}^{2}}{Y_{1}}-\frac{\varphi_{2}^{2}}{Y_{\mathrm{P}}}\right)$.

Appelons $\alpha_{0}$ la vitesse caractéristique de la plaque :

$$
\alpha_{0}^{2}=\frac{\Phi_{0}^{2}}{\mu_{0} \bar{\omega} e l^{2} X} .
$$

Il vient :

$$
\begin{aligned}
v^{2} & =\alpha_{0}^{2} \frac{\varphi_{1}^{2}}{x_{1}} \cdot \frac{X}{X_{2}} \cdot \frac{\left(X_{\mathrm{P}}-X_{1}\right)\left(X_{2}-X_{\mathrm{P}}\right)}{X_{\mathrm{P}}\left(X-X_{\mathrm{P}}\right)} \\
& =\alpha_{0}^{2} \frac{\varphi_{2}^{2}}{y_{2}} \cdot \frac{X}{Y_{1}} \cdot \frac{\left(Y_{1}-Y_{\mathrm{P}}\right)\left(Y_{\mathrm{P}}-Y_{2}\right)}{Y_{\mathrm{P}}\left(X-Y_{\mathrm{P}}\right)} .
\end{aligned}
$$

On peut en déduire en principe le mouvement de la plaque $X_{\mathrm{P}}(t)$. En fait, les expressions sont trop compliquées pour être intégrables analytiquement de façon directe. On peut cependant faire quelques approxima- tions dans le cas où $X_{2}-X_{1} \gg X_{1}, Y_{2}$ et où, par suite, $X_{2} \simeq Y_{1} \simeq X$. On a tout d'abord:

$$
\left(\frac{\varphi_{1}}{\varphi_{2}}\right)^{2}=\frac{X_{1} X_{2}}{Y_{1} Y_{2}} \simeq \frac{X_{1}}{Y_{2}}=\frac{x_{1}}{y_{2}}
$$

et

$$
\alpha_{0}^{2} \frac{\varphi_{1}^{2}}{x_{1}}=\alpha_{0}^{2} \frac{\varphi_{2}^{2}}{y_{2}}=\alpha^{2} .
$$

Le mouvement de la plaque peut alors se décrire comme suit :

- Pour $X_{\mathrm{P}} \simeq X_{1}$, c'est-à-dire à l'accélération de la plaque :

$$
v^{2} \simeq \alpha^{2} \frac{X_{\mathrm{P}}-X_{1}}{X_{\mathrm{P}}}
$$

En appelant $t_{1}$ le temps écoulé depuis le départ, ceci s'intègre en :

$\sqrt{\frac{X_{\mathrm{P}}}{X_{1}}\left(\frac{X_{\mathrm{P}}}{X_{1}}-1\right)}+\operatorname{Arg} \operatorname{sh} \sqrt{\frac{X_{\mathrm{P}}}{X_{1}}-1}=\frac{\alpha t_{1}}{X_{1}}$.

- Pour $Y_{\mathrm{P}} \simeq Y_{2}$, c'est-à-dire au ralentissement de la plaque :

$$
v^{2} \simeq \alpha^{2} \frac{Y_{\mathrm{P}}-Y_{2}}{Y_{\mathrm{P}}}
$$

qui s'intègre en :

$\sqrt{\frac{Y_{\mathrm{P}}}{Y_{2}}\left(\frac{Y_{\mathrm{P}}}{Y_{2}}-1\right)}+\operatorname{Arg} \operatorname{sh} \sqrt{\frac{Y_{\mathrm{P}}}{Y_{2}}-1}=\frac{\alpha t_{2}}{Y_{2}}$

$t_{2}$ étant le temps nécessaire jusqu'à l'arrêt de la plaque.

Les lois (15) et (16) décrivant l'accélération et le ralentissement de la plaque sont donc les mêmes. Elles déterminent complètement $X_{\mathrm{P}} / X_{1}$ en fonction de $\alpha t_{1} / X_{1}$ et $Y_{\mathrm{P}} / Y_{2}$ en fonction de $\alpha t_{2} / Y_{2}$ (Fig. 8) et l'on a, pour des valeurs identiques de $X_{\mathrm{P}} / X_{1}$ et $Y_{\mathrm{P}} / Y_{2}$ :

$$
\frac{t_{1}}{t_{2}}=\frac{X_{1}}{Y_{2}}=\frac{x_{1}}{y_{2}} .
$$

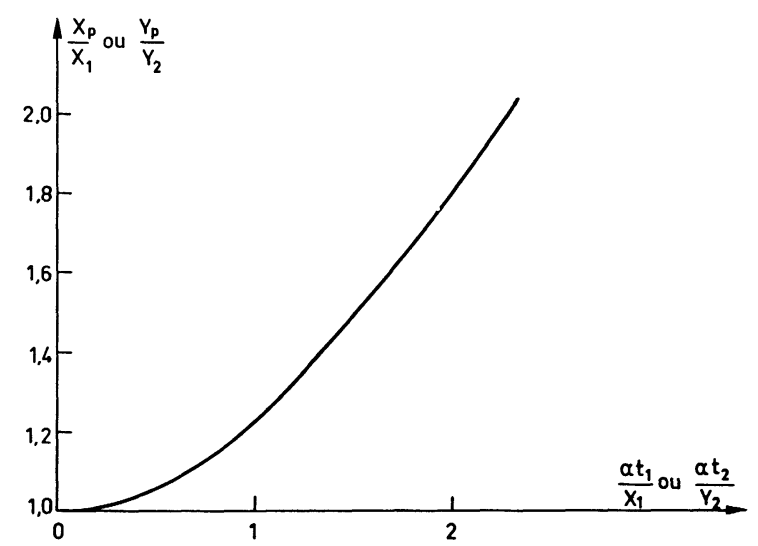

Fig. 8. - Accélération $\left(X_{\mathrm{p}} / X_{1}\right)$ et ralentissement $\left(Y_{\mathrm{p}} / Y_{2}\right)$ de la plaque en fonction du temps. 
Nous appellerons $t_{\mathrm{a}}$ et $t_{\mathrm{r}}$ les durées d'accélération et de ralentissement correspondant à $X_{\mathrm{P}}=2 X_{1}$ et $Y_{\mathrm{P}}=2 Y_{2} \cdot t_{\mathrm{a}}$ représente aussi le temps d'extraction de l'énergie de la bobine $\mathfrak{B}_{1}$ et $t_{\mathrm{r}}$ le temps de remplissage de la bobine $\mathfrak{B}_{2}$. Nous avons :

$$
t_{\mathrm{a}}=2,3 \frac{X_{1}}{\alpha}, \quad t_{\mathrm{r}}=2,3 \frac{Y_{2}}{\alpha} .
$$

Si l'on désire que $t_{\mathrm{r}}$ soit court, il faut donc que $y_{2}$ soit petit.

Entre ces deux périodes, c'est-à-dire pour

$$
2 X_{1} \leqslant X_{\mathrm{P}} \leqslant X-2 Y_{2},
$$

la vitesse varie lentement de $\alpha / \sqrt{2}$ à $\alpha_{M}$ (valeur maximale de la vitesse, obtenue à partir de (12) avec $\left.X_{\mathrm{P}}=\varphi_{1} X\right)$ puis revient à $\alpha / \sqrt{2}$. Avec les mêmes approximations que précédemment, on a :

$$
\alpha_{M}^{2}=\alpha^{2}\left(1-\frac{x_{1}}{\varphi_{1}}\right) .
$$

On admettra que le mouvement est quasi uniforme, de vitesse $\alpha^{\prime} \sim 0,8$ à $0,9 \alpha$.

La figure 9 représente le mouvement effectif de la plaque dans le cas particulier $x_{1}=0,12$ et $y_{2}=0,04$. Nous voyons qu'il est parfaitement justifié d'admettre un mouvement uniforme entre l'accélération et le ralentissement ; la valeur de la vitesse correspond bien.

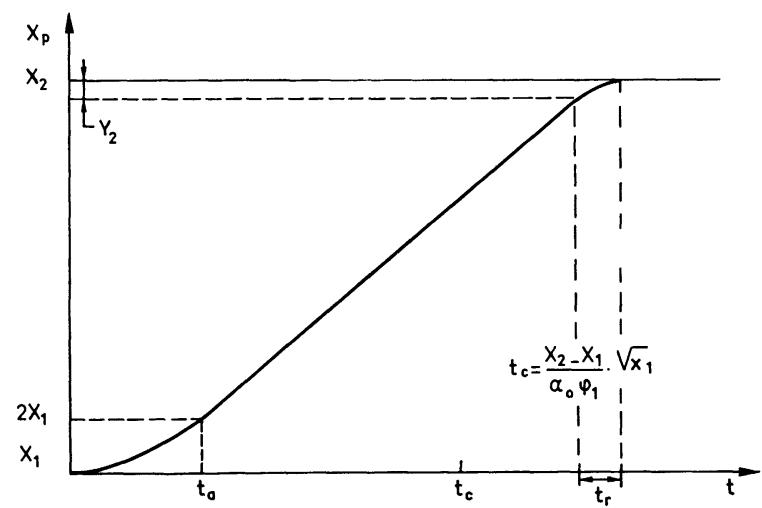

FIG. 9. - Mouvement effectif de la plaque pour $x_{1}=0,12$ et $y_{2}=0,04$. Le temps caractéristique est donné par

$$
t_{\mathrm{c}}=\left(X_{2}-X_{1}\right) / \alpha_{0} \varphi_{1} x_{1}^{-1 / 2} \text {. }
$$

Remarquons que, pour que cette description du mouvement et en particulier celle de l'accélération soit correcte, il faut que la montée du champ $B_{1 \mathbf{i}}$ au niveau de la plaque et par suite l'ouverture du disjoncteur mécanique $\mathrm{D}$ se fasse en un temps au plus égal à $X_{1} / \alpha$. Nous poserons :

$$
t=\frac{X_{1}}{\alpha}=\frac{t_{\mathrm{a}}}{2,3}=\frac{x_{1}}{y_{2}} \cdot \frac{t_{\mathrm{r}}}{2,3} .
$$

Nous en déduisons le rapport entre les temps d'ouverture de l'absorbeur et de l'ouvreur :

$$
\frac{t_{\mathrm{r}}}{\tau}=\frac{2,3 y_{2}}{x_{1}} \text {. }
$$

5. Rendement en présence des pertes liées à la conductivité non infinie du système. - 5.1 Notations. - Pour cette étude, précisons tout d'abord les notations en nous reportant à la figure 10. Les flux

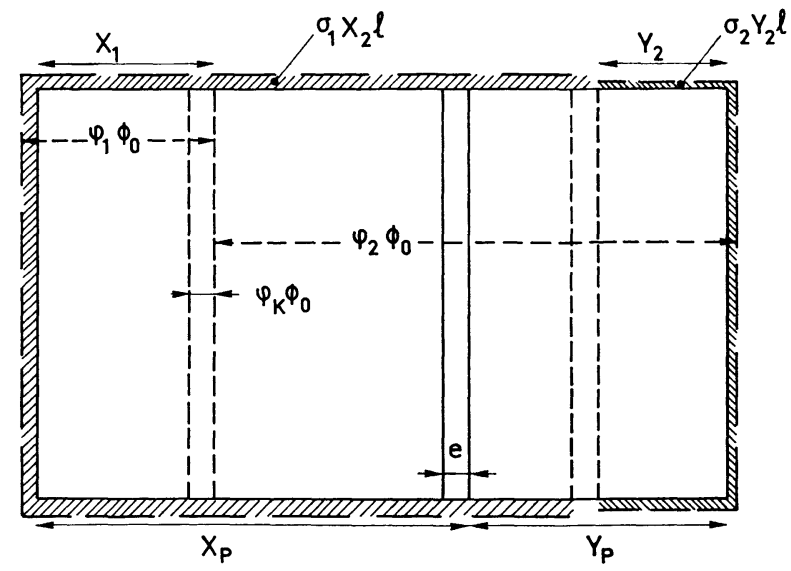

Fig. 10. - Schéma de l'absorbeur montrant les flux piégés.

sont maintenant en partie dans les épaisseurs de peau et dans la plaque et, désormais :

$-\varphi_{1} \Phi_{0}$ comprend le flux situé dans la plaque et le flux à gauche de $\mathrm{P}$, y compris dans les épaisseurs de peau.

- $\varphi_{2} \Phi_{0}$ représente tout le flux à droite de $\mathrm{P}$.

- $X_{\mathrm{P}}$ comprend l'épaisseur de la plaque.

En ce qui concerne les épaisseurs de peau, elles interviennent par la surface qu'elles occupent, en particulier à l'arrêt de la plaque. Remarquons que du côté de $\mathfrak{B}_{2}$, c'est-à-dire du côté de la récupération de l'énergie, cette surface est nettement plus grande dans le montage à une plaque que dans celui à deux (Fig. 1 et 2); la fraction de champ piégé est donc plus importante. Nous ne considérerons que le second montage et nous poserons :

$\sigma_{1}=$ surface de la peau à gauche de $\mathrm{P} / X_{2} l$

$\sigma_{2}=$ surface de peau de la bobine $\Re_{2} /$ surface $S_{2}$ de $\mathfrak{B}_{2}$.

Si $r_{2}$ est le rayon de $\mathcal{B}_{2}$ et $\delta_{2}$ son épaisseur de peau, on a $S_{2}=\pi r_{2}^{2}=Y_{2} l$ et

$$
\sigma_{2}=\frac{2 \pi r_{2} \delta_{2}}{\pi r_{2}^{2}}=\frac{2 \sqrt{\pi}}{\sqrt{Y_{2} l}} \delta_{2} .
$$

Si l'épaisseur de la plaque est inférieure aux épaisseurs de peau, le courant qui y circule est uniformément réparti et le champ magnétique varie linéairement à l'intérieur entre les deux valeurs $B_{1}$ et $B_{2}$. Au départ $B_{2 \mathrm{i}} \ll B_{1 \mathrm{i}}$, le flux piégé dans $P, \varphi_{k} \Phi_{0}$ est donné par :

$$
\varphi_{k} \simeq \frac{e}{2 X_{1}} \varphi_{1}=k \varphi_{1}
$$


A la fin, $B_{2 \mathrm{i}} \gg B_{1 \mathrm{i}}, \varphi_{k}^{\prime}$ vaut :

$$
\varphi_{k}^{\prime} \simeq \frac{e}{2 Y_{2}} \varphi_{2}=k^{\prime} \varphi_{2}
$$

et l'énergie piégée dans la plaque

$$
W_{k}=\frac{e}{3 Y_{2}} \cdot \frac{\varphi_{2}^{2} \Phi_{2}^{2}}{2 \mu_{0} l Y_{2}} .
$$

5.2 ENERGIE UTILISABLE. - L'énergie récupérée, qui valait

$$
W_{\mathrm{u}}=\frac{\Phi_{0}^{2} \varphi_{2}^{2}}{2 \mu_{0} l Y_{2}}
$$

est modifiée par plusieurs causes :

- Par suite de la diffusion du champ magnétique au travers de $P$, les flux deviennent $\varphi_{2} \Phi_{0}\left(1+p_{2}\right)$ dans $\Re_{2}$ et $\left(\varphi_{1}-\Phi_{k}\right) \Phi_{0}\left(1+p_{1}\right)$ à gauche de $\mathrm{P}$. Le flux global restant conservatif, on a :

$$
\varphi_{1}(1-k)\left(1+p_{1}\right) \Phi_{0}+k^{\prime} \varphi_{2} \Phi_{0}+\varphi_{2}\left(1+p_{2}\right) \Phi_{0}=\Phi_{0}
$$

ou encore

$$
\left(p_{1}-k\right) \varphi_{1}+\left(p_{2}+k^{\prime}\right) \varphi_{2}=0 .
$$

- La distance d'arrêt devient :

$$
Y_{2}^{\prime}=Y_{2}(1+\eta)
$$

par suite de la variation des flux et des pertes d'énergie par effet joule dans $\mathrm{P}, W_{\mathrm{JP}}$, et dans les parois de l'enceinte, $W_{\mathrm{Je}}$. On posera :

$$
W_{\mathrm{J}}=W_{\mathrm{JP}}+W_{\mathrm{Je}} .
$$

$Y_{2}^{\prime}$ (ou $\eta$ ) se calcule à partir de la conservation de l'énergie totale en remarquant que les flux se répartissent comme suit : $\varphi_{1} \Phi_{0}\left(1+p_{1}-k\right)$ sur la surface $\left[X_{2}\left(1+\sigma_{1}\right)-\eta Y_{2}-e\right] l$ et $\varphi_{2} \Phi_{0}\left(1+p_{2}\right)$ sur la surface $Y_{2}\left(1+\eta+\sigma_{2}\right) l$. Nous avons donc :

$$
\begin{array}{r}
\frac{\Phi_{0}^{2}}{2 \mu_{0} l}\left[\frac{\varphi_{1}^{2}\left(1+2 p_{1}-2 k\right)}{X_{2}\left(1+\sigma_{1}\right)-\eta Y_{2}-e}+\frac{\varphi_{2}^{2}\left(1+2 p_{2}\right)}{Y_{2}\left(1+\eta+\sigma_{2}\right.}\right]+ \\
+W_{k}+W_{\mathrm{J}}=W_{0}
\end{array}
$$

soit :

$$
\begin{aligned}
& \frac{\varphi_{1}^{2}}{X_{1}}\left(2 p_{1}-2 k-\sigma_{1}+\eta \frac{Y_{2}}{X_{2}}+\frac{e}{X_{2}}\right)+ \\
& \quad+\frac{\varphi_{2}^{2}}{Y_{2}}\left(2 p_{2}-\eta-\sigma_{2}+\frac{2 k^{\prime}}{3}\right)=-\frac{2 \mu_{0} l}{\varphi_{0}^{2}} W_{\mathrm{J}} .
\end{aligned}
$$

Avec les mêmes hypothèses que précédemment (cf. (13)) :

$$
\begin{aligned}
\frac{\varphi_{1}^{2}}{X_{2}}(- & 2 k+e)+\frac{2 k^{\prime}}{3} \cdot \frac{\varphi_{2}^{2}}{Y_{2}} \simeq \frac{\varphi_{2}^{2}}{Y_{2}} \times \\
& \times\left(-\frac{e}{X_{2}}+e \frac{X_{1}}{X_{2}}+\frac{e}{3 Y_{2}}\right) \simeq \frac{\varphi_{2}^{2}}{Y_{2}} \cdot \frac{e}{3 Y_{2}} .
\end{aligned}
$$

De même, compte tenu de (26) et de ce, qu'en général, $\varphi_{2} \lesssim \varphi_{1}$

$$
\begin{aligned}
\frac{\varphi_{1}^{2}}{X_{2}}\left(2 p_{1}-2 k\right)+\frac{\varphi_{2}^{2}}{Y_{2}}\left(2 p_{2}\right. & \left.+2 k^{\prime}\right) \simeq \\
& \simeq\left(2 p_{2}+2 k^{\prime}\right) \frac{\varphi_{2}^{2}}{Y_{2}}
\end{aligned}
$$

et

$$
\eta\left(\frac{\varphi_{1}^{2} Y_{2}}{X_{2}^{2}}-\frac{\varphi_{2}^{2}}{Y_{2}}\right) \simeq-\eta \frac{\varphi_{2}^{2}}{Y_{2}}
$$

(29) pourra donc être simplifié.

- Enfin l'énergie transférée n'est pas totalement utilisable puisqu'une partie reste piégée dans l'épaisseur de peau de $\mathfrak{B}_{2}$. L'énergie récupérable vaut:

$$
\begin{aligned}
W_{\mathrm{u}}+\Delta W_{\mathrm{u}} & =\frac{\varphi_{2}^{2} \Phi_{0}^{2}\left(1+2 p_{2}\right)}{2 \mu_{0} l Y_{2}\left(1+\eta+\sigma_{2}\right)} \cdot\left(1-\sigma_{2}\right) \\
& =W_{\mathrm{u}}\left(1+2 p_{2}-\eta-2 \sigma_{2}\right) .
\end{aligned}
$$

Compte tenu de (29), (30), (31) et (32), ceci s'écrit :

$$
\begin{aligned}
\frac{\Delta W_{\mathrm{u}}}{W_{\mathrm{u}}} & =2 p_{2}-\eta-2 \sigma_{2} \\
& =-\frac{W_{\mathrm{J}}}{W_{\mathrm{u}}}-\frac{e}{3 Y_{2}}+\frac{X_{1}}{X} \sigma_{1}-\sigma_{2} .
\end{aligned}
$$

Pour obtenir $\Delta W_{\mathbf{u}}$, il nous faut évaluer les pertes joules et les épaisseurs de peau. Il n'est pas utile de connaître l'importance de la diffusion des flux au travers de $P$, si ce n'est en ordre de grandeur pour vérifier qu'elle reste faible.

5.3 Epaisseurs de PEAU. - Appelons $\rho_{\mathrm{e}}$ la résistivité des bobines et des parois de l'absorbeur et $\rho$ celle de la plaque.

$\mathrm{Au}$ cours de l'accélération, entre les temps $t_{1}=0$ et $t_{1} \simeq 2 X_{1} / \alpha, X_{\mathrm{P}}$ varie de $X_{1}$ à $2 X_{1}$ (cf. Fig. 8) et l'induction $B_{1}$ qui varie comme $1 / X_{\mathrm{P}}$, est divisée par deux en variant de façon quasi linéaire :

$$
B_{1} \simeq \frac{\varphi_{1} \Phi_{0}}{X_{1} l}\left(1-\frac{\alpha t_{1}}{4 X_{1}}\right) \simeq \frac{\varphi_{1} \Phi_{0}}{X_{1} l} \mathrm{e}^{-\mathrm{t} / \tau_{1}} .
$$

On peut alors admettre que l'épaisseur de peau est la même que pour un courant exponentiel :

$$
\delta_{1}=\sqrt{\frac{\rho_{\mathrm{e}} \tau_{1}}{\mu_{0}}}=2 \sqrt{\frac{\rho_{\mathrm{e}} X_{1}}{\mu_{0} \alpha}}
$$

dans les parois à gauche de $\mathrm{P}$

$$
\delta_{1}^{\prime}=2 \sqrt{\frac{\rho X_{1}}{\mu_{0} \alpha}}
$$

dans la plaque.

Au cours du mouvement uniforme à la vitesse $\alpha^{\prime} \lesssim \alpha$, $B_{1}$ décroît un petit peu plus vite que suivant (34) et $\delta_{1}$ et $\delta_{1}^{\prime}$ deviennent un peu plus petits. Enfin au 
cours du ralentissement, $B_{1}$ décroît très peu. $\delta_{1}$ et $\delta_{\mathrm{P}}$ caractériseront bien les effets de peau pour $B_{1}$.

De même, les effets de peau relatifs à $B_{2}$ seront caractérisés par :

$$
\delta_{2}^{\prime}=2 \sqrt{\frac{\rho Y_{2}}{\mu_{0} \alpha}}
$$

dans la plaque

$$
\delta_{2}=2 \sqrt{\frac{\rho_{\mathrm{e}} Y_{2}}{\mu_{0} \alpha}}
$$

dans les parois à droite de $\mathbf{P}$, ces valeurs étant valables surtout pour le ralentissement.

5.4 ECHAuffement DE LA PLAQUE. - Cet échauffement se calcule par :

$$
\bar{\omega} c \theta=\int \rho i^{2} d t
$$

où $\bar{\omega}$ et $c$ sont les masses et chaleur spécifiques de $P$. Nous supposons toujours la plaque suffisamment fine $\left(e<\delta_{1}^{\prime}\right.$ et $\left.\delta_{2}^{\prime}\right)$ pour que la densité de courant $i$ y soit uniforme ; nous négligeons les modifications des inductions liées à la diffusion au travers de $P$. Enfin, nous choisissons des inductions $B_{1}$ et $B_{2}$ de même sens, de façon que $i$ soit petit et par suite $\theta$ faible (Il peut en être facilement ainsi si le flux de commande est créé par une source extérieure ; il en est ainsi s'il est créé par diffusion du flux initial mais pas si l'on utilise une enceinte extérieure). Nous avons alors :

$$
i=\frac{B_{1}-B_{2}}{\mu_{0} e} .
$$

Pendant l'accélération,

$$
B_{1}=\frac{\varphi_{1} \Phi_{0}}{X_{\mathrm{P}} l} \gg B_{2} .
$$

L'échauffement est alors majoré par $\theta_{\mathrm{a}}$ :

$$
c \theta_{\mathrm{a}}=\frac{\rho \alpha}{\mu_{0} e} \int \frac{X_{1}^{2}}{X_{\mathrm{P}}^{2}} \mathrm{~d}\left(\frac{\alpha t_{1}}{X_{1}}\right) .
$$

Compte tenu de (15) qui donne $X_{1} / X_{\mathrm{P}}$ en fonction de $\alpha t_{1} / X_{1}$, on peut intégrer (40) numériquement pour $1<X_{1} / X_{\mathrm{P}}<2$; on trouve :

$$
c \theta_{\mathrm{a}}=1,4 \frac{\rho \alpha}{\mu_{0} e} .
$$

De même, on obtient au ralentissement :

$$
c \theta_{\mathrm{r}}=1,4 \frac{\rho \alpha}{\mu_{0} e}
$$

Pendant le mouvement uniforme :

$$
c \theta_{\mathrm{u}}=\frac{\rho}{\mu_{0} e} \alpha_{0}^{2} \int\left(\frac{\varphi_{1}^{2}}{x_{\mathrm{P}}^{2}}-\frac{2 \varphi_{1} \varphi_{2}}{x_{\mathrm{P}} y_{\mathrm{P}}}+\frac{\varphi_{2}^{2}}{y_{\mathrm{P}}^{2}}\right) \mathrm{d} t .
$$

Avec $x_{\mathrm{P}}=\alpha^{\prime} t$, il vient en intégrant de $x_{\mathrm{P}}=2 x_{1}$ à $x_{\mathrm{P}}=1-2 y_{2}$ et en utilisant les hypothèses déjà faites (cf. (13)) :

$$
\begin{aligned}
c \theta_{u} & \simeq \frac{\rho \alpha_{0}^{2}}{\mu_{0} e \alpha^{\prime}}\left(\frac{\varphi_{1}^{2}}{x_{1}}+2 \varphi_{1} \varphi_{2} \log 4 x_{1} y_{2}\right) \\
& \simeq \frac{\rho \alpha^{2}}{\mu_{0} e \alpha^{\prime}}\left(1+2 \sqrt{x_{1} y_{2}} \log 4 x_{1} y_{2}\right) .
\end{aligned}
$$

En pratique cela donne $c \theta_{\mathrm{u}} \simeq 0(\sqrt{x} \log x \sim-1$ pour $0,02<x<0,16)$.

Finalement, l'échauffement est sensiblement donné par :

$$
c \theta \simeq \frac{3 \rho \alpha}{\mu_{0} e}
$$

Le rapport entre l'énergie joule dépensée et l'énergie utile vaut alors :

$$
\frac{W_{\mathrm{JP}}}{W_{\mathrm{u}}} \simeq \frac{W_{\mathrm{JP}}}{W_{\mathrm{s}}}=\frac{2 c \theta}{\alpha^{2}}=\frac{6 \rho}{\mu_{0} e \alpha}=\varepsilon .
$$

Cette évaluation de $W_{\mathrm{JP}}$ suppose $e<\delta_{1}^{\prime}$ et $\delta_{2}^{\prime}$. En général, $Y_{2}$ est plus petit que $X_{1}$ et $\delta_{2}^{\prime}$ est un peu plus petit que $\delta_{1}^{\prime}$ mais peu. Aussi, si l'on a $e \lesssim \delta_{1}^{\prime}$ et $e \gtrsim \delta_{2}^{\prime}$, le courant reste réparti de façon à peu près homogène même au ralentissement et le calcul reste valable. Si $e>\delta_{1}^{\prime}$, l'échauffement sera du même ordre que pour $e \sim \delta_{1}^{\prime}$ mais il restera superficiel.

Nous avons aussi admis que la résistivité $\rho$ était constante c'est-à-dire que $\theta$ était très petit. S'il n'en est pas ainsi, l'échauffement réel $\theta_{\mathrm{f}}$ sera tel que :

$$
c \theta_{0} \log \frac{\theta_{\mathrm{f}}+\theta_{0}}{\theta_{0}}=c \theta \simeq \frac{3 \rho \alpha}{\mu_{0} e} .
$$

Le tableau I donne la correspondance entre $\theta$ et $\theta_{\mathrm{f}}$.

$\begin{array}{llllllllll}\theta & 86 & 122 & 153 & 182 & 208 & 232 & 254 & 275 & 294\end{array}$

$\begin{array}{llllllllll}\theta_{\mathrm{f}} & 100 & 150 & 200 & 250 & 300 & 350 & 400 & 450 & 500 .\end{array}$

5.5 ECHAUfFement DES PAROIS. - Cet échauffement a lieu dans les épaisseurs de peau.

Considérons le montage à deux plaques et supposons que la hauteur $l$ de l'enceinte soit très supérieure à la course $X_{2}-X_{1}$ de la plaque, ce qui sera en général le cas (cf. § 7). L'énergie joule perdue dans les parois de l'enceinte le sera alors essentiellement dans la zone hachurée de la figure 2, parcourue par le courant $i_{1}$ et d'épaisseur $\delta_{1}$. Or $B_{1}$, qui détermine $i_{1}$, est donné tout d'abord par (34) puis s'écarte un peu de cette loi. Cependant, comme l'échauffement a surtout lieu lorsque $i_{1}$ est fort, c'est-à-dire pendant l'accélération, on aura une très bonne approximation de l'échauffement superficiel global $\theta_{\mathrm{e}}$ en écrivant qu'il est égal à celui provoqué par le champ exponentiel (34) pour $t$ variant de 0 à l' $\infty$. Si les constantes physiques de la paroi sont $\rho_{\mathrm{e}}, \bar{\omega}_{\mathrm{e}}, c_{\mathrm{e}}$, on a :

$$
\bar{\omega}_{\mathrm{e}} c \theta_{\mathrm{e}}=\frac{1}{2 \mu_{0}} \cdot \frac{\varphi_{1}^{2} \Phi_{0}^{2}}{X_{1}^{2} l^{2}} \text {. }
$$


L'énergie joule dépensée est alors concentrée dans une épaisseur de peau $\delta_{1} / 2$ et vaut :

$$
W_{\mathrm{Je}}=\frac{1}{2 \mu_{0}} \frac{\varphi_{1}^{2} \Phi_{0}^{2}}{X_{1}^{2} l^{2}} \frac{\delta_{1}}{2} l=W_{\mathrm{s}} \sqrt{\frac{\rho_{\mathrm{e}}}{\mu_{0} \alpha X_{1}}} .
$$

Si on pose

$$
e=k \delta_{1}^{\prime} \quad(k \lesssim 1)
$$

on a :

$$
\frac{W_{\mathrm{JP}}}{W_{\mathrm{Je}}}=\frac{3}{k} \sqrt{\frac{\rho}{\rho_{\mathrm{e}}}} .
$$

Si la plaque et l'enceinte sont en même métal ou si $\rho \gg \rho_{\mathrm{e}}, W_{\mathrm{JP}} \gg W_{\mathrm{Je}}$, les pertes d'énergie par effet joule se situent principalement dans la plaque.

5.6 Diffusion du Champ Magnétique. - Pour étudier la diffusion au travers de $P$, nous supposons que les flux gelés dans les épaisseurs de peau et dans la plaque sont négligeables. Nous posons que, lorsque la plaque est en $X_{\mathrm{P}}$, les flux utiles valent $\varphi_{1}\left(1+q_{1}\right)$ et $\varphi_{2}\left(1+q_{2}\right)\left(q_{1}=p_{1}\right.$ et $q_{2}=p_{2}$ pour $\left.X_{\mathrm{P}}=X_{2}\right)$. On établit facilement les équations :

$$
\frac{\rho}{\mu_{0} e}\left(\frac{\varphi_{1}}{X_{\mathrm{P}}}-\frac{\varphi_{2}}{Y_{\mathrm{P}}}\right)=-\varphi_{1} \frac{\mathrm{d} q_{1}}{\mathrm{~d} t}=+\varphi_{2} \frac{\mathrm{d} q_{2}}{\mathrm{~d} t} .
$$

Leur intégration entre $X_{1}$ et $Y_{2}$ donne :

$$
\begin{gathered}
p_{1}=1,8 \frac{\rho}{\mu_{0} e \alpha}\left(-1+\sqrt{\frac{y_{2}}{x_{1}}}\right) \\
=0,3 \varepsilon\left(-1+\sqrt{\frac{y_{2}}{x_{1}}}\right) \\
p_{2}=1,8 \frac{\rho}{\mu_{0} e \alpha}\left(\frac{x_{1}}{y_{2}}-1\right)=0,3 \varepsilon\left(\sqrt{\frac{x_{1}}{y_{2}}}-1\right) .
\end{gathered}
$$

5.7 Perte sur l'énergie utilisable. - Compte tenu des diverses expressions établies dans ce paragraphe 5, on a :

$$
\frac{\Delta W_{\mathrm{u}}}{W_{\mathrm{u}}}=-\frac{W_{\mathrm{J}}}{W_{\mathrm{u}}}-\frac{e}{3 Y_{2}}+\frac{X_{1}}{X} \sigma_{1}-\sigma_{2}
$$

ou

$$
\begin{aligned}
\frac{\Delta W_{\mathrm{u}}}{W_{\mathrm{u}}}=-\frac{6 \rho}{\mu_{0} e \alpha}-\sqrt{\frac{\rho_{\mathrm{e}}}{\mu_{0} \alpha X_{1}}}-\frac{e}{3 Y_{2}}+ \\
+\sqrt{\frac{\rho_{\mathrm{e}}}{\mu_{0} \alpha}}\left(\frac{2 X_{1}^{3 / 2}}{X}-4 \sqrt{\frac{\pi}{l}}\right)
\end{aligned}
$$

(dans le calcul de $\sigma_{1}$, nous avons supposé que

$$
\left.X_{2}-X_{1} \ll l\right) \text {. }
$$

Or,

$$
\frac{2 X_{1}^{3 / 2}}{X}-4 \sqrt{\frac{\pi}{l}} \ll \frac{1}{\sqrt{X_{1}}},
$$

le flux gelé dans les parois de $\mathfrak{B}_{1}$ et de l'absorbeur et celui gelé dans les parois de $\mathfrak{B}_{2}$ entraînent une perte négligeable comparée à celle par l'effet [Joule dans les parois, il reste donc :

$$
\Delta W_{\mathrm{u}} \simeq-\left(W_{\mathrm{J}}+W_{k}\right) .
$$

L'énergie utilisable perdue est de l'ordre de grandeur de l'énergie joule dépensée dans le système augmentée de l'énergie gelée dans la plaque. Finalement :

$$
\frac{\Delta W_{\mathrm{u}}}{W_{\mathrm{u}}} \simeq-\frac{6 \rho}{\mu_{0} e \alpha}-\frac{e}{3 Y_{2}}-\sqrt{\frac{\rho_{\mathrm{e}}}{\mu_{0} \alpha X_{1}}} .
$$

Le troisième terme étant éventuellement négligeable (cf. (48)).

6. Pertes dues aux défauts des plaques. - Une plaque n'est jamais d'épaisseur rigoureusement constante. Son épaisseur est en fait comprise entre les valeurs $e\left(1+\frac{d}{2}\right)$ et $e\left(1-\frac{d}{2}\right)$. Pour une fabrication tout à fait courante $d=10^{-2}$; pour une plus soignée, nous admettrons $d=3 \times 10^{-3}$. A l'arrêt des plaques l'une contre l'autre, certaines parties viennent en contact avec une vitesse nulle, d'autres ont déjà rebondi et d'autres ne sont pas encore arrivées. Pour éviter au maximum de coincer du flux, un isolant fin est placé entre les deux plaques. Il subsiste cependant une surface non nulle avec du flux non récupérable. Nous allons l'évaluer.

A un instant quelconque, toutes les portions des plaques sont soumises aux mêmes inductions,magnétiques $\mathbf{B}_{1}$ et $\mathbf{B}_{2}$ donc à la même densité de force $\mathbf{f}$. Or on a, pour un élément quelconque d'épaisseur locale $e$ :

$$
\int_{t_{0}}^{t} \mathbf{f} \mathrm{d} t=\bar{\omega} e \int_{t_{0}}^{t} \mathrm{~d} v=\bar{\omega} e\left[v(t)-v\left(t_{0}\right)\right]=\bar{\omega} e v(t)
$$

$\int_{t_{0}}^{t} \mathrm{f} \mathrm{d} t$ est une quantité parfaitement définie, indépendante de l'épaisseur de la plaque. A l'instant $t$, les vitesses $v$ et $v^{\prime}$ des éléments d'épaisseur ' $e$ et ' $e^{\prime}$ sont donc telles que :

$$
\frac{v}{v^{\prime}}=\frac{e}{e^{\prime}}
$$

En particulier, les vitesses de tous les éléments s'annulent en même temps. Autrement dit, tous les éléments de la plaque s'arrêtent au même instant $t_{\mathrm{f}}$ mais „pas au même endroit ; la plaque est déformée de

$$
\pm \Delta L=\frac{d}{2}\left(X_{2}-X_{1}\right)
$$

En fait, certaines parties, les plus fines, ayant déjà rebondi, la déformation est différente.

Considérons donc ce rebondissement et évaluons tout d'abord l'importance de la déformation par 
rapport à l'épaisseur de la plaque. On a $e=k \delta_{1}^{\prime}$ avec $k \lesssim 1$; compte tenu de (36) et (44), il vient :

$$
\frac{e}{\Delta L}=k^{2} \frac{\varepsilon}{3 d} \cdot \frac{X_{1}}{X_{2}-X_{1}}
$$

$k^{2}$ est de l'ordre de $1, \varepsilon$ de quelques $\%, d$ de l'ordre de $1 \%, \frac{X_{1}}{X_{2}-X_{1}} \ll 1$; il en résulte $\frac{e}{\Delta L}<1$. L'épaisseur de la plaque est inférieure à l'ordre de grandeur des déformations.

Calculons maintenant la vitesse de la plaque alors qu'il lui reste encore une distance $z=Y_{\mathrm{P}}-Y_{2}$ à parcourir avant son arrêt et supposons $z \lesssim \Delta L \ll Y_{2}$. D'après (16), on a :

$$
2 \sqrt{z Y_{2}}=\alpha t_{2}
$$

et

$$
v(z)=\alpha \sqrt{\frac{z}{Y_{2}}} .
$$

Lorsque les premiers points des plaques se touchent, $z=\Delta L$.

$$
v(\Delta L)=\alpha \sqrt{\frac{d}{2 y_{2}}}
$$

est nettement inférieur à $\alpha$ car $d \lesssim 1 \%$ et $y_{2} \gtrsim$ quelques $\%$ ). Si $\alpha$ est de l'ordre de quelques $\mathrm{km} / \mathrm{s}, v(\Delta L)$ et à fortiori $v(z<\Delta L)$ sera nettement inférieur à la vitesse du son dans la plaque conductrice $(\sim 5 \mathrm{~km} / \mathrm{s})$. Comme de plus $e \lesssim \Delta L$, le temps nécessaire à la propagation d'un choc dans la plaque sera bien plus petit que celui nécessaire à l'arrêt de cette plaque ; l'approximation adiabatique sera valable et les rebondissements auront lieu de façon quasi instantanée, la plaque reprenant le mouvement symétrique de celui avec lequel elle arrivait.

Les parties en avance et celles en retard des deux plaques se présentent les unes en face des autres de façon aléatoire. Nous admettrons que tout se passe comme si chacune rebondissait sur une paroi fixe placée dans le plan de symétrie (Fig. 11). Si $t^{\prime}$ est l'avance temporelle prise par les éléments plus fins que l'épaisseur moyenne, nous voyons qu'au temps

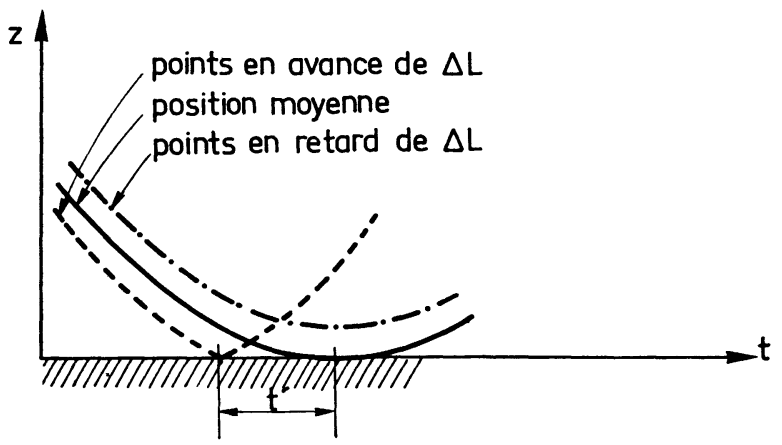

FIG. 11. - Rebondissement d'une plaque sur une paroi rigide. $t^{\prime} / 2$ avant l'arrêt, la surface perdue pour le flux passe par un minimum (cf. Fig. 12). Nous l'évaluerons pour chaque plaque de hauteur $l / 2$ à $\frac{\Delta L . l}{4}$. A cet
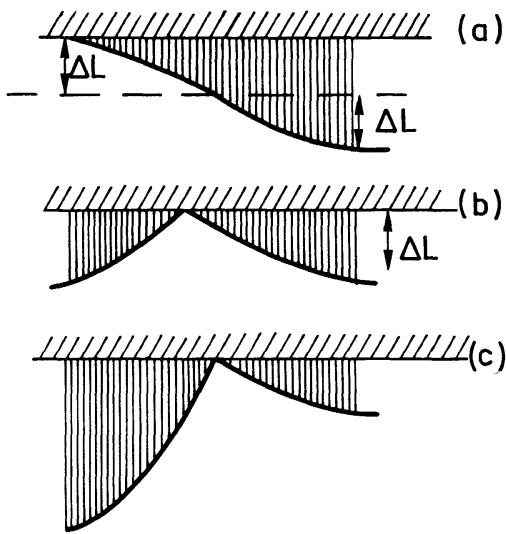

Fig. 12. - Evolution de la forme de la plaque en fonction du temps. (a) Les premiers points touchent la paroi. (b) Temps $t^{\prime} / 2$

(c) Temps $t^{\prime}$. La surface perdue pour le flux est hachurée.

instant, l'induction magnétique $\boldsymbol{B}_{2}^{\prime}$ est inférieure à $B_{2}$; elle vaut (en négligeant les autres pertes) :

$$
B_{2}^{\prime}=\frac{\varphi_{2} \Phi_{0}}{\left(Y_{2}+\frac{\Delta L}{2}\right) l}
$$

et l'énergie récupérable devient :

$$
W_{\mathrm{u}}^{\prime}=\frac{B_{2}^{\prime 2}}{2 \mu_{0}} Y_{2} l=W_{\mathrm{u}}\left(1-\frac{\Delta L}{Y_{2}}\right)=W_{\mathrm{u}}\left(1-\frac{d}{2 y_{2}}\right) \text {. }
$$

On peut se demander s'il n'est pas préférable de prévoir la séquence de telle sorte que l'arrêt des plaques se fasse dans la position (a) de la figure 12 ; alors la distance d'arrêt du système vaut

$$
Y_{2}^{\prime \prime}=Y_{2}+\Delta L \text {. }
$$

Pour cela, il faut partir d'un flux $\varphi_{2}^{\prime \prime} \Phi_{0}$ légèrement supérieur à $\varphi_{2} \Phi_{0}$. On peut alors voir que ce fonctionnement est un peu moins favorable puisque :

$$
W_{\mathrm{u}}^{\prime \prime}=W_{\mathrm{u}}\left(1-\frac{3}{2} \frac{\Delta L}{Y_{2}}\right)
$$

Nous avons négligé les défauts locaux. Nous admettrons que, par suite de la rigidité mécanique, ils ont peu d'effet.

7. Performances globales du système. - Le rendement global est finalement donné par :

$r_{\mathrm{f}}=1-x_{1}-y_{2}-\frac{d}{2 y_{2}}-\frac{e}{3 Y_{2}}-\varepsilon-\sqrt{\frac{\rho_{\mathrm{e}}}{\mu_{0} X_{1} \alpha}}$ 
$1-x_{1}-y_{2}$ représente le rendement $r$ en absence de pertes (cf. (8)), $d / 2 y_{2}$ mesure l'influence des défauts des plaques, $e / 3 Y_{2}$ tient compte du flux gelé dans les plaques et les deux derniers termes de l'effet Joule dans les plaques et les parois.

7.1 Choix Des PlaQues. - Il s'agit de préciser leur épaisseur par rapport à $\delta_{1}^{\prime}$ et de voir s'il est préférable de les faire en cuivre ou en aluminium. Pour cela nous allons voir l'évolution des divers paramètres caractéristiques du montage $\left(W_{\mathrm{s}}, e, X, \varepsilon, \alpha\right)$. Nous fixerons l'échauffement $\theta$ de la plaque et le temps d'accélération $t_{\mathrm{a}}$; nous supposerons que la hauteur $l$ et la largeur $\mathcal{L}$ du montage sont telles que :

$$
\begin{aligned}
& l=\lambda X \\
& \mathcal{L}=\mu X
\end{aligned}
$$

et aussi que

$$
\begin{array}{rlll}
e=k k^{\prime} \delta_{\mathrm{a}} & \left(\text { si } e<\delta_{1}^{\prime},\right. & k<1, & \left.k^{\prime}=1\right) \\
& \left(\text { si } e>\delta_{1}^{\prime},\right. & k=1, & \left.k^{\prime}>1\right) .
\end{array}
$$

Nous rappellerons enfin que le temps d'ouverture du disjoncteur placé entre la bobine $\mathfrak{B}_{1}$ et l'absorbeur vaut (cf. (21)).

$$
\tau=\frac{t_{\mathrm{a}}}{2,3}=\frac{x_{1}}{2,3 y_{2}} t_{\mathrm{r}}=\frac{x_{1}}{y_{2}} \tau_{\mathrm{r}}
$$

Il vient, après quelques calculs et en appelant $W_{\mathrm{s}}$ l'énergie stockée par unité de volume de $\mathfrak{B}_{1}$.

$$
\begin{aligned}
e & =2 k k^{\prime} \sqrt{\frac{\rho \tau}{\mu_{0}}} \\
X_{1} & =\frac{2}{3} \frac{k}{k^{\prime}} \sqrt{\frac{\mu_{0}}{\rho}} c \theta \tau^{3 / 2} \\
\varepsilon & =\frac{k^{\prime}}{k^{2}} \frac{9 \rho}{2 \mu_{0} c \theta \tau} \\
\alpha & =\frac{k}{k^{\prime}} \frac{2 c \theta}{3} \sqrt{\frac{\mu_{0} \tau}{\rho}} \\
w_{\mathrm{s}} & =\frac{2}{3} k^{2} \bar{\omega} \mathrm{c} \theta \\
\frac{e}{3 Y_{2}} & =k^{\prime 2} \frac{\rho}{\mu_{0} c \theta \tau} \cdot \frac{x_{1}}{y_{2}} \\
W_{\mathrm{s}} & =\frac{k^{5}}{k^{\prime 3}} \frac{16 \lambda \mu}{81 x_{1}^{2}}\left(\frac{\mu_{0}}{\rho}\right)^{3 / 2} \bar{\omega}(c \theta)^{4} \tau^{9 / 2} .
\end{aligned}
$$

On voit que si $k$ est pris inférieur à 1 , les pertes joule s'accroissent, l'énergie par unité de volume diminue et l'énergie totale décroît encore plus. Il faut donc choisir $k=1$. Si $k^{\prime}$ devient plus grand que 1 , l'énergie par unité de volume reste constante, les dimensions et l'énergie totale diminuent, les pertes dues à l'effet joule et au flux gelé dans $P$ s'accroissent. On a donc tout intérêt à prendre $k=k^{\prime}=1$, ce que nous ferons désormais.
Comparons maintenant les performances obtenues avec des plaques en aluminium et en cuivre. On a pour les mêmes $\theta$ et $\tau$ :

$$
\begin{gathered}
\frac{e_{\mathrm{Al}}}{e_{\mathrm{Cu}}}=1,25 ; \quad \frac{X_{1 \mathrm{Al}}}{X_{1 \mathrm{Cu}}}=\frac{\alpha_{\mathrm{Al}}}{\alpha_{\mathrm{Cu}}}=1,88 ; \\
\frac{\varepsilon_{\mathrm{Al}}}{\varepsilon_{\mathrm{Cu}}}=\frac{\left(e / 3 Y_{2}\right)_{\mathrm{Al}}}{\left(e / 3 Y_{2}\right)_{\mathrm{Cu}}}=0,67 ; \\
\frac{w_{\mathrm{s}(\mathrm{Al})}}{w_{\mathrm{s}(\mathrm{Cu})}}=0,72 ; \quad \frac{W_{\mathrm{s}(\mathrm{Al})}}{W_{\mathrm{s}(\mathrm{Cu})}}=4,82 .
\end{gathered}
$$

Avec l'aluminium, les pertes sont plus faibles et l'énergie totale plus élevée mais les dimensions sont plus grandes. Il semble que l'aluminium soit préférable, mais on peut, dans certains cas, lorsque l'énergie mise en jeu n'est pas trop grande, préférer le cuivre. Nous prendrons maintenant des plaques en aluminium. L'énergie transférée par unité de volume de plaque est :

$$
w_{\mathrm{p}}=r_{\mathrm{f}} W_{\mathrm{s}} / e \lambda \mu X^{2}=r_{\mathrm{f}} \bar{\omega} \alpha^{2} / 2=r_{\mathrm{f}} \bar{\omega} c \theta / \varepsilon
$$

Il sera intéressant de comparer $w_{\mathrm{p}}$ à la densité d'énergie $w_{\mathrm{e}}$ transférable par une explosion de feuille. On sait que, pour l'aluminium, une telle explosion absorbe $25 \mathrm{~kJ} / \mathrm{cm}^{3}$ et que, dans le meilleur des cas c'est-à-dire pour des inductances de stockage et de charge égales, l'énergie absorbée est deux fois plus importante que l'énergie transférée. On a donc $w_{\mathrm{e}} \leqslant 12,5 \mathrm{~kJ} / \mathrm{cm}^{3}$.

Notons enfin que les pertes joule dans les parois de l'enceinte, qui entraînent une diminution du rendement égale à (cf. (48)) :

$$
\sqrt{\frac{\rho_{\mathrm{e}}}{\mu_{0} X_{1} \alpha}}=\frac{1}{3} \sqrt{\frac{\rho_{\mathrm{e}}}{\rho} \varepsilon}
$$

seront minimales si $\rho_{\mathrm{e}}$ est minimal, c'est-à-dire si l'enceinte est en cuivre. Dans ce cas, les pertes joule totales vaudront :

$$
\varepsilon_{\mathrm{J}} \simeq 1,3 \varepsilon
$$

7.2 UTILISATION DU MONTAGE. - Nous constatons qu'une augmentation de $\theta$ diminue les pertes et accroît l'énergie par unité de volume et encore plus l'énergie totale. Pour préciser les performances possibles de l'absorbeur, nous choisirons donc $\theta$ le plus grand possible. Nous prendrons $\theta=250^{\circ}$ ce qui correspond à une augmentation effective de la température de $400^{\circ}$ (cf. Tableau I).

Finalement, avec: une enceinte en cuivre, des plaques en aluminium d'épaisseur égale à l'épaisseur de peau, un échauffement $\theta_{\mathrm{f}} \simeq 400^{\circ}\left(\theta \simeq 250^{\circ}\right)$, on a si $\tau$ est en $\mu$ s :

$$
\begin{aligned}
& e_{\mathrm{mm}}=0,3 \sqrt{\tau} ; X_{1 \mathrm{~mm}}=1,02 \tau^{3 / 2} ; \varepsilon_{\mathrm{J}}=0,57 / \tau ; \\
& \alpha_{\mathrm{km} / \mathrm{s}}=1,02 \sqrt{\tau} ; w_{\mathrm{s}}=0,41 \mathrm{~kJ} / \mathrm{cm}^{3} ; B_{1}=32 \mathrm{~T} ; \\
& e / 3 Y_{2}=0,1 x_{1} / y_{2} \tau ; W_{\mathrm{s}(\mathrm{J})}=0,44 \lambda \mu x_{1}^{-2} \tau^{9 / 2}
\end{aligned}
$$




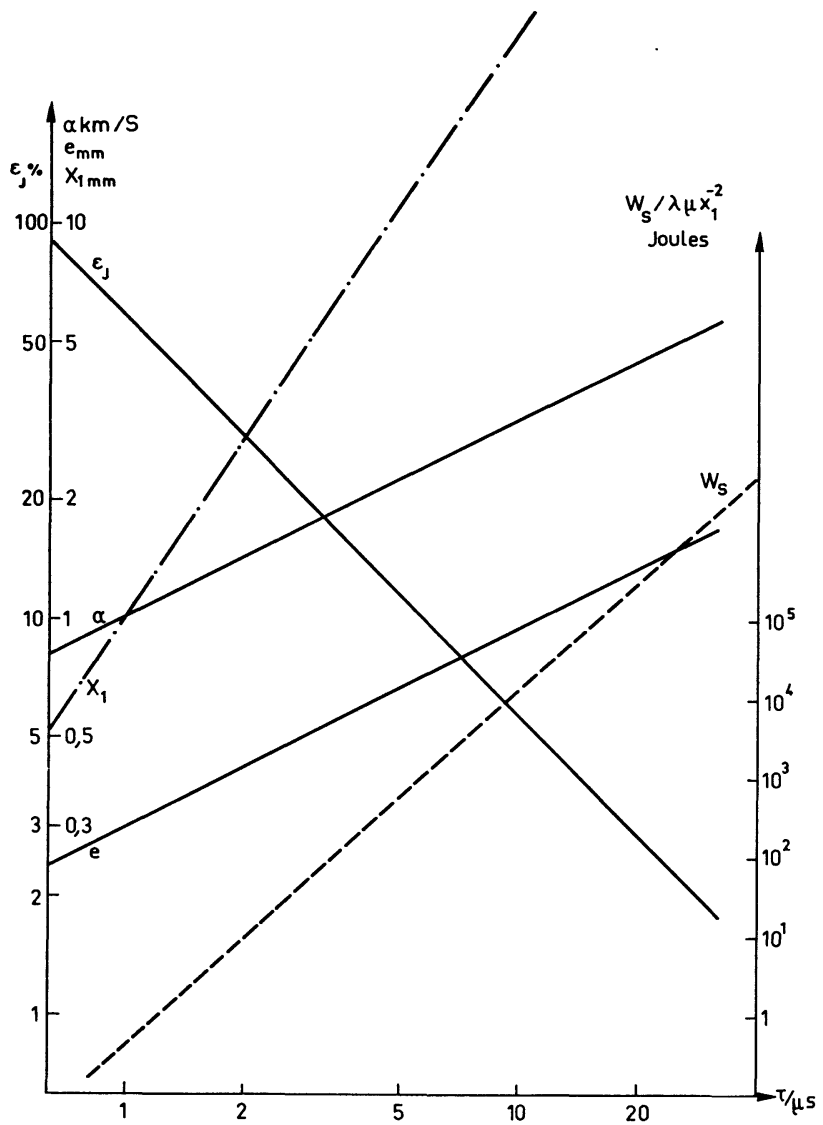

Fig. 13. - Variations en fonction de $\tau$ de divers paramètres de l'absorbeur pour $\theta=250^{\circ}\left(\theta_{\mathrm{f}}=400^{\circ}\right)$. Les plaques sont en aluminium, les parois en cuivre. et

$r_{\mathrm{f}}=1-x_{1}-y_{2}-\frac{d / 2+0,1 x_{1} / \tau}{y_{2}}-\frac{0,57}{\tau}$.

Certaines de ces expressions sont représentées figure 13 en fonction de $\tau$.

Pour connaître les possibilités effectives de l'absorbeur, il nous faut maintenant choisir $x_{1}$ et $y_{2}$. Plusieurs considérations entrent en jeu, plus ou moins contradictoires :

- Le rendement $r_{\mathrm{f}}$ doit être élevé ; $x_{1}$ doit donc être petit. Quant à $y_{2}$, il doit être tel que

$$
y_{2}-\left(d / 2+0,1 x_{1} \tau^{-1}\right) / y_{2}
$$

soit minimum. Si $0,1 x_{1} \tau^{-1} \ll d$, cela donne

$$
y_{2} \simeq \sqrt{d / 2}
$$

- La fraction d'énergie dépensée pour la commande $C=y_{2} / 1-y_{2}$ doit être petite, ce qui implique que $y_{2}$ le soit.

- Le rapport $x_{1} / 2,3 y_{2}$, qui représente le facteur de raccourcissement du temps de délivrance de l'énergie, doit être grand et par suite $x_{1}$ grand et $y_{2}$ petit.

Le tableau II montre l'influence du choix de $y_{2}$ sur les pertes (lorsque $0,1 x_{1} \tau^{-1} \ll d$ ) et sur $C$. On voit qu'il est bon de prendre $y_{2} \simeq 0,7 \sqrt{d / 2}$ car cela diminue notablement l'énergie de commande et le temps de délivrance de l'énergie tout en augmentant à peine les pertes. Ce tableau montre aussi l'extrême intérêt de plaques très régulières :

TABLEAU II

$\begin{array}{cccccc}d & 10^{-2} & 10^{-2} & 3 \times 10^{-3} & 3 \times 10^{-3} & 3 \times 10^{-3} \\ y_{2} & 0,07(=\sqrt{d / 2}) & 0,05 & 0,04(=\sqrt{d / 2}) & 0,03 & 0,02 \\ \mathrm{C} \% & 7,5 & 5,3 & 4 & 3,1 & 2,0 \\ \left(y_{2}+\frac{d}{2 y_{2}}\right) \% & 14 & 15 & 7,8 & 8,0 & 9,5\end{array}$

\begin{tabular}{|c|c|c|c|c|c|c|c|c|c|c|c|c|c|}
\hline \multicolumn{2}{|c|}{$\begin{array}{r}d \\
\theta_{\mathrm{f}}=400 \mathrm{~K} \\
\end{array}$} & $y_{2}$ & $\begin{array}{c}d / 2 y_{2} \\
\%\end{array}$ & $\begin{array}{l}C \\
\%\end{array}$ & $x_{1}$ & $\begin{array}{c}\tau \\
\mu \mathrm{s}\end{array}$ & $\begin{array}{c}t_{\mathrm{r}} \\
\mu \mathrm{S}\end{array}$ & $\begin{array}{l}r_{\mathrm{f}} \\
\%\end{array}$ & $\begin{array}{c}e \\
\mathrm{~mm}\end{array}$ & $\begin{array}{c}X_{1}-X_{2} \\
\mathrm{~mm}\end{array}$ & $\stackrel{w_{\mathrm{p}}}{\mathrm{kJ} / \mathrm{cm}^{3}}$ & $\begin{array}{l}W_{\mathrm{s}} \\
\mathrm{MJ}\end{array}$ & $\begin{array}{c}\alpha \\
\mathrm{km} / \mathrm{s}\end{array}$ \\
\hline & & - & - & - & - & - & - & - & - & - & - & - & - \\
\hline \multirow[t]{3}{*}{ (a) } & $10^{-2}$ & 0,05 & 10 & 5,3 & 0,12 & 5 & 4,8 & 58 & 0,67 & 79 & 4,0 & $0,13-4,3$ & 2,3 \\
\hline & & & & & & 10 & 9,6 & 66 & 0,95 & 223 & 9,3 & 2,9-97 & 3,2 \\
\hline & & & & & & 20 & 19 & 70 & 1,34 & 631 & 20 & $66-2200$ & 4,5 \\
\hline \multirow[t]{4}{*}{ (b) } & $3 \times 10^{-3}$ & 0,03 & 5 & 3 & 0,12 & 5 & 2,9 & 61 & 0,67 & 81 & 4,0 & $0,13-4,3$ & 2,3 \\
\hline & & & & & & 10 & 5,8 & 71 & 0,95 & 229 & 9,4 & 2,9-97 & 3,2 \\
\hline & & & & & & 20 & 11,5 & 76 & 1,34 & 646 & 20 & $66-2200$ & 4,5 \\
\hline & & & & & & 100 & 58 & 79 & 3 & 7220 & 105 & $9 \times 10^{4}-3 \times 10^{6}$ & 10 \\
\hline \multirow[t]{3}{*}{ (c) } & $3 \times 10^{-3}$ & 0,03 & 5 & 3 & 0,06 & 5 & 5,8 & 71 & 0,67 & 173 & 4,7 & $0,5-17$ & 2,3 \\
\hline & & & & & & 10 & 11,5 & 79 & 0,95 & 489 & 10,5 & $11,6-386$ & 3,2 \\
\hline & & & & & & 20 & 23 & 22 & 1,34 & 1384 & 22 & $262-8700$ & 4,5 \\
\hline
\end{tabular}

TABleaU III

$\theta=25^{\circ}$

$\begin{array}{lllllllll}\text { (d) } 3 \times 10^{-3} & 0,03 & 5 & 3 & 0,12 & 100 & 58 & 71\end{array}$

722

0,9

9-300 
Le tableau III donne quelques exemples typiques de l'utilisation de l'absorbeur. Les limites d'énergie indiquées correspondent à $\lambda=1, \mu=3$ qui représentent des valeurs plutôt minimales et $\lambda=\mu=10$ qui peuvent être augmentées si besoin. Nous constatons que le rendement de transfert est bon. Il est d'autant meilleur

- que $\tau$ est grand,

- que $d$ est petit.

Tous les exemples correspondent à $y_{2} \simeq 0,7 \sqrt{d / 2}$. Les énergies $W_{\mathrm{s}}$ mises en jeu sont parfois fort élevées, surtout pour $\tau \simeq 100 \mu$ s. Pour les réduire, il suffit d'abaisser $\theta$. C'est ce que nous avons fait en $(d)$; comme $\tau$ est grand, le rendement reste bon.

L'épaisseur des plaques est de l'ordre du $\mathrm{mm}$. L'énergie transférée par unité de volume de plaque est très comparable à celle transférée par une feuille explosante, donc la masse des plaques est du même ordre que celle des feuilles qui permettraient d'obtenir la même énergie dans la charge (à partir d'une énergie initiale au moins deux fois plus grande). Les dimensions du système sont très raisonnables. Par exemple, un absorbeur de $2,8 \mathrm{~m} \times 1,4 \mathrm{~m} \times 44 \mathrm{~cm}$ comportant deux plaques de $0,95 \mathrm{~mm}$ d'épaisseur, permet d'obtenir à partir de $100 \mathrm{MJ}$, lorsqu'il est associé à un disjoncteur ouvrant en $10 \mu \mathrm{s}, 60 \mathrm{MJ}$ délivrés en $12 \mu \mathrm{s}$ si les plaques sont définies à $1 \%$ et 66 MJ délivré en $6 \mu$ s pour des plaques définies à $3 \times 10^{-3}$. La masse des plaques, un peu plus grande dans le premier cas, est environ $50 \%$ supérieure à celle des feuilles explosées.

8. Conclusion. - Associé à un disjoncteur mécanique rapide, l'absorbeur décrit permet de transférer l'énergie d'une inductance vers une autre avec un rendement excellent (60-70\%) bien supérieur au rendement maximal habituel $(25 \%)$ obtenu pour les systèmes statiques (feuilles explosées par exemple). Il est bien adapté aux transferts d'énergie de l'ordre de $10 \mathrm{MJ}$ ou plus s'effectuant en un temps de l'ordre de $10 \mu \mathrm{s}$. L'énergie transférée par unité de volume de plaque $\left(\sim 10 \mathrm{~kJ} / \mathrm{cm}^{3}\right)$ pour l'aluminium est tout à fait comparable à celle d'une feuille explosée équivalente. Les destructions à chaque fonctionnement sont limitées à deux plaques et il n'y a pas de choc direct sur les parois. Cette simplicité est à comparer à l'utilisation difficile des feuilles explosées qui nécessite des surfaces plus grandes (facteur 10), un matériau de bourrage et des enceintes nettement plus résistantes aux chocs. Cet absorbeur paraît donc un progrès considérable dans la maîtrise des transferts rapides de hautes énergies électromagnétiques.

\section{Bibliographie}

[1] Rıoux, C., Conditions d'amorçage d'une réaction thermonucléaire dans un plasma chauffé par l'implosion rapide d'une enceinte matérielle. Rapport interne LF 13, nov. 73.

[2] Rioux, C., JABlon, C., Nucl. Fus. 15 (1975) 425.

[3] Velikhov, E. P., Vedinov, A. A., Bogdanets, A. D., Golubev, V. S., KasharskiI, E. G., Sov. Phys. Tech. Phys. 18 (1973) 274.

[4] Robson, A. E., Linus an approach to controlled fusion through the use of Megagauss Magnetic Fields. Report of NRL Progress. June (1973), p. 7.
[5] Rioux, C., Sultanem, F., Etude théorique des machines asynchrones sans fer à rotor massif LF 50 (octobre 1975).

[6] Guillet, R., Hahn, R., Lucidarme, J., Rioux, C., Rev. Phys. Appl. 8 (1973) 53.

[7] Robson, A. E., Turchi, P., Lupton, W., URY, M., WarNICK, W., Proceedings of the International Conference on Energy Storage, Compression and Switching (Nov. 74) (Edité par Plenum-Press New York and London) 1976 p. 95.

[8] Rioux, C., Rioux-Damidau, F., Rev. Phys. Appl. 9 (1975) 539.

[9] Benford, J., Calvin, H., Smith, I., Aslin, H., Ref. [7] p. 39. 